\title{
The Two-Layer Structure of the Entrainment Zone in the Convective Boundary Layer
}

\author{
Jade Rachele Garcia And Juan Pedro Mellado \\ Max Planck Institute for Meteorology, Hamburg, Germany
}

(Manuscript received 20 May 2013, in final form 10 February 2014)

\begin{abstract}
The entrainment zone (EZ) of a dry, shear-free convective boundary layer growing into a linearly stratified fluid is studied by means of direct numerical simulation. The scale separation between the boundary layer thickness and the Kolmogorov length scale is shown to be sufficient to observe Reynolds number similarity in the statistics of interest during the equilibrium entrainment regime. Contrary to previous considerations, the vertical structure of the entrainment zone is found to be better described by the superposition of two sublayers: 1) an upper EZ sublayer that is dominated by overshooting thermals and is characterized by a penetration depth that scales with the ratio of the convective velocity and the buoyancy frequency of the free troposphere and 2) a lower EZ sublayer that is dominated by troughs of mixed fluid and is characterized by the integral length scale of the mixed layer. Correspondingly, different buoyancy scales are identified. The consequences of this multiplicity of scales on the entrainment rate parameters are evaluated directly, without resorting to any bulk model, through an exact relation among the mean entrainment rate, the local buoyancy increment, and both the turbulent and the finite-thickness contributions to the entrainment ratio $A$ measured at the height of minimum buoyancy flux. The smaller turbulent contribution to $A$ that is usually observed for relatively thick EZs is found to be compensated by the smaller local buoyancy increment instead of by the finite-thickness contribution. The two-layer structure of the entrainment zone is found to affect the exponent of the power-law relation between the normalized mean entrainment rate and the convective Richardson number such that the exponent deviates from -1 for typical atmospheric conditions, although it asymptotically approaches -1 for higher Richardson numbers.
\end{abstract}

\section{Introduction}

The zero-order bulk model (ZOM) (Zilitinkevich 1991) predicts well enough the mean entrainment rate of a dry, shear-free convective boundary layer (CBL) with constant surface buoyancy flux growing into a linearly stratified fluid. However, the ZOM cannot predict accurately the parameters that affect the mean entrainment rate (which we call entrainment rate parameters), such as the entrainment ratio and the buoyancy increment of CBLs with relatively thick entrainment zone (EZ) (Sullivan et al. 1998; Fedorovich et al. 2004a). Such limitation stems from the ZOM's representation of the $\mathrm{EZ}$ as an infinitesimally thin layer. Extensions of the basic zero-order bulk model have been developed, such as first-order models (Betts 1974; vanZanten et al. 1999) and general structure models (Fedorovich and Mironov

Corresponding author address: Jade Rachele Garcia, Max Planck Institute for Meteorology, Bundesstrasse 53, 20146 Hamburg, Germany.

E-mail: jade.garcia@mpimet.mpg.de
1995; Fedorovich et al. 2004a). However, incomplete understanding of the entrainment zone has led to difficulty in modeling the evolution of entrainment rate parameters, as reflected by the disagreement among the different parameterizations proposed in the literature (Deardorff et al. 1980; Sorbjan 1999; Fedorovich et al. 2004a; Träumner et al. 2011).

In this study, we consider a CBL growing into a linearly stratified free troposphere [see Fedorovich et al. (2004a) for a review] to investigate the possible selfsimilar behavior of the buoyancy profiles inside the entrainment zone (Fedorovich and Mironov 1995; Sorbjan 1999; Fedorovich et al. 2004a) and the characteristic scales associated with such behavior. We also assess the effect of this vertical structure on the entrainment rate parameters (Betts 1974; Sullivan et al. 1998) and the relation between the entrainment rate and Richardson number (Deardorff et al. 1980; Fedorovich et al. 2004a; Träumner et al. 2011).

The definition of the entrainment rate parameters and the relation among them remain problematic for complex bulk models that try to incorporate the effect of the 
EZ vertical structure (see, e.g., Betts 1974; Fedorovich and Mironov 1995; Sullivan et al. 1998). As a case in point, the arbitrary definition of the upper limit of the entrainment zone affects both the finite-thickness contribution to the entrainment ratio, and the relation between the normalized mean entrainment rate and the convective Richardson number, as defined in Sullivan et al. (1998) and Fedorovich et al. (2004a). We therefore perform the analysis directly on the mean entrainment rate equation that is derived from the mean buoyancy transport equation, without imposing any bulk model assumption on the vertical profiles of mean buoyancy and mean buoyancy flux. An analysis based on the actual profiles can help to eliminate part of the uncertainty and arbitrariness in those definitions. In particular, we show in this paper that the two-layer structure that we observe in the entrainment zone explains part of the disagreement among previous works.

Most of the studies that addressed these issues were based on data from large-eddy simulations (LES), which, as explained by Sullivan et al. (1998) and Fedorovich et al. (2004a), were at that time not highly resolved and are unavoidably tainted by uncertainty due to subgrid-scale models. In this respect, resolving more of the small-scale turbulence should help address the limitations of LES (Stevens and Lenschow 2001). However, Sullivan and Patton (2011) show that, even when the resolution has been increased significantly, there is still significant uncertainty at the entrainment zone due to subgrid-scale models, in particular for the potential temperature variance. These observations motivate the use of direct numerical simulation (DNS) in our analysis. Despite the fact that DNS can only achieve Reynolds numbers significantly lower than typical atmospheric values, DNS has become a very valuable tool in turbulence research during the last decades because it eliminates the uncertainty introduced by a turbulence model (Moin and Mahesh 1998; Jimenez 2013). The use of DNS is further motivated and justified by Reynolds number similarity: the principle, based on observation, that some statistics become independent of the Reynolds number once this is large enough (Tennekes and Lumley 1972; Dimotakis 2000; Monin and Yaglom 2007). From this proposition, the questions that naturally arise are "What is large enough for a particular configuration?" and "How do different statistics evolve toward such an asymptotic behavior?". The results presented here show that the Reynolds numbers we achieve are sufficiently high to gain new insight into the entrainment zone of an atmospheric CBL growing into a linearly stratified free troposphere.

We structure the paper as follows. After describing the formulation in section 2 , we present the dimensional analysis in section 3 . In section 4 , we summarize the different definitions of the height of the CBL top, which are used for the discussion in the following sections, and show that the analysis presented in this paper corresponds to the equilibrium (quasi-steady) entrainment regime (Fedorovich et al. 2004a). We then focus on the structural analysis of the entrainment zone in sections 5 and 6 , where we consider first its vertical structure, and then evaluate the effect of this vertical structure on the entrainment rate parameters.

\section{Formulation}

We use the Navier-Stokes equations in the Boussinesq approximation

$$
\begin{aligned}
\boldsymbol{\nabla} \cdot \mathbf{v} & =0, \\
\frac{\partial \mathbf{v}}{\partial t}+\nabla \cdot(\mathbf{v} \otimes \mathbf{v}) & =-\nabla p+\nu \nabla^{2} \mathbf{v}+b \mathbf{k}, \\
\frac{\partial b}{\partial t}+\nabla \cdot(\mathbf{v} b) & =\kappa \nabla^{2} b,
\end{aligned}
$$

where $\mathbf{v}(\mathbf{x}, t)$ is the velocity vector with components $\left(v_{1}, v_{2}, w\right)$ along the directions $O x_{1}, O x_{2}$, and $O z$, respectively; $p$ is a modified pressure divided by the constant reference density; and $b(\mathbf{x}, t)$ is the buoyancy [which can be related to, e.g., the virtual potential temperature $\theta_{v}$ by a linear relation, $b=g\left(\theta_{v}-\theta_{v, 0}\right) / \theta_{v, 0}$, where $\theta_{v, 0}$ is the reference value]. The parameter $\nu$ is the kinematic viscosity, $\kappa$ is the molecular diffusivity, and $\mathbf{k}$ is the unit vector along $O z$. The system is statistically homogeneous over the horizontal planes and the statistics depend only on the vertical distance from the surface $z$ and time $t$.

A no-penetration, no-slip boundary condition is used at the bottom plate and a no-penetration, free-slip boundary condition at the top. Neumann boundary conditions are used for the buoyancy at the top and the bottom to maintain fixed constant fluxes. In addition, the velocity and buoyancy fields are relaxed toward zero and the background buoyancy profile,

$$
b_{0}(z)=N^{2} z,
$$

respectively, inside a sponge layer occupying the upper region of the computational domain. Preliminary simulations (not shown) have been used to adjust the height of the top boundary so that it is far enough from the turbulent region to avoid any significant interaction. Periodicity is used at the sides.

Parameters of the two simulations considered in the study are summarized in Table 1 . The size of the computational grid is $5120 \times 5120 \times 840$ for the reference case, which is denoted as Re100. Stretching is used in the vertical direction to increase the resolution near the 
TABLE 1. Simulation properties. Columns $4-8$ provide data at the final time of the simulations. The convective Reynolds number Re $\mathrm{R}_{*}$ is defined by Eq. (8) and the turbulent Reynolds number $\mathrm{Re}_{t}=e^{2} /(\varepsilon \nu)$, where $e$ is the turbulence kinetic energy and $\varepsilon$ its viscous dissipation rate, is the maximum value within the CBL. The Kolmogorov scale $\eta=\left(\nu^{3} / \varepsilon\right)^{1 / 4}$ is the minimum value within the CBL. The length scale $\delta$ is defined by Eq. (21). The last three columns give the time-averaged values (beyond $z_{\text {end }} / L_{0}{ }^{\prime} \simeq 10$ ) of the root-mean-square (rms) of the turbulent fluctuations taken at the height of maximum $w_{\text {rms }}$.

\begin{tabular}{|c|c|c|c|c|c|c|c|c|c|c|}
\hline Simulation & Grid & $\mathrm{Re}_{0}$ & $\mathrm{Re}_{t}$ & $\mathrm{Re}_{*}$ & $z_{\text {end }} / L_{0}$ & $z_{\text {end }} / \eta$ & $\delta / L_{0}$ & $w_{\mathrm{rms}} / w_{*}$ & $\boldsymbol{v}_{1, \mathrm{rms}} / w_{*}$ & $b_{\mathrm{rms}} / b_{*}$ \\
\hline Re100 & $5120 \times 5120 \times 840$ & 117 & 2860 & 5480 & 18 & 490 & 1.3 & 0.71 & 0.42 & 1.25 \\
\hline $\operatorname{Re} 040$ & $2560 \times 2560 \times 704$ & 42 & 1600 & 3160 & 26 & 320 & 1.6 & 0.69 & 0.41 & 1.26 \\
\hline
\end{tabular}

surface and to move the top boundary farther up, so that the domain size is $215 L_{0} \times 215 L_{0} \times 56.6 L_{0}$, where $L_{0}$ is a reference length scale that is defined in the following section. The simulation is terminated when the boundary layer thickness is about $18 L_{0}$. At the final time, the aspect ratio of the horizontal domain size and the CBL thickness is around 12:1. The second simulation, $\mathrm{Re} 040$, in Table 1 with a reference Reynolds number $\mathrm{Re}_{0}$ (to be defined later) roughly a third of that in Re100 has been used to study the effect of $\operatorname{Re}_{0}$ and to achieve a deeper CBL, with thickness up to $26 L_{0}$. The horizontal size of the computational domain in Re040 is the same as in Re100.

Discretization of Eq. (1) in space is performed using sixth-order spectral-like compact finite differences on a structured Cartesian grid. A low-storage fourth-order accurate Runge-Kutta scheme is used for time stepping. The discrete solenoidal constraint is satisfied to machine accuracy using a Fourier decomposition along the periodic horizontal planes $x_{1} O x_{2}$ and a factorization of the resulting set of equations along the vertical coordinate (Mellado and Ansorge 2012). The local ratio between the vertical grid spacing, $\Delta z$, and the Kolmogorov scale, $\eta=$ $\left(\nu^{3} / \varepsilon\right)^{1 / 4}$ (where $\varepsilon$ is the viscous dissipation rate), is $\Delta z / \eta \simeq$ 1.2 or less. Appendix $\mathrm{C}$ demonstrates that this grid resolution is already enough for the results discussed in this paper to become independent of the grid resolution.

\section{Dimensional analysis}

We focus in this paper on the fully developed turbulent regime that is established after the initial transient, when the initial conditions have been sufficiently forgotten (Tennekes and Lumley 1972; Monin and Yaglom 2007), so that the parameter space $\left\{\nu, \kappa, B_{0}, N\right\}$ defines the system completely. Following Zilitinkevich (1991) and Fedorovich et al. (2004a), we choose $N$ and $B_{0}$ to nondimensionalize the problem, which yields a reference time scale $N^{-1}$ and a reference length scale

$$
L_{0}=\left(B_{0} / N^{3}\right)^{1 / 2}
$$

The length scale $L_{0}$ can be interpreted as an Ozmidov scale $\left(\varepsilon / N^{3}\right)^{1 / 2}$, which is a measure of the smallest eddy size affected by a background stratification $N^{2}$ in a turbulent field characterized by a viscous dissipation rate $\varepsilon$ (see e.g., Ozmidov 1965; Smyth and Moum 2000). In the CBL, the viscous dissipation rate is, approximately, an order-of-one fraction of the surface buoyancy flux $B_{0}$; as we will show later, the direct influence of $N^{2}$ is concentrated in the entrainment zone. Therefore, this reference Ozmidov scale $L_{0}$ can be interpreted as an estimate for an integral length scale of turbulence inside the entrainment zone, or a region therein; the results discussed in section 5 support this argument. (An additional interpretation of $L_{0}$ as the minimum CBL thickness is presented in appendix A.)

The system depends only on two nondimensional parameters: a reference buoyancy Reynolds number

$$
\operatorname{Re}_{0}=\frac{B_{0}}{\nu N^{2}}
$$

and a Prandtl number $\operatorname{Pr}=\nu / \kappa$. This work investigates the role of $\mathrm{Re}_{0}$ only, so we fix $\operatorname{Pr}=1$. The reference buoyancy Reynolds number, $\mathrm{Re}_{0}$, is often used in the study of the interaction between turbulence and stable stratification (see, e.g., Hebert and de Bruyn Kops 2006; Chung and Matheou 2012). It is constructed using $L_{0}$ as a length scale and $\left(L_{0} B_{0}\right)^{1 / 3}$ as a velocity scale, where the latter follows from inertial-range Kolmogorov scaling and the viscous dissipation rate being proportional to $B_{0}$. Hence, according to the interpretation of $L_{0}$ as the local integral scale of turbulence inside the entrainment zone, $\mathrm{Re}_{0}$ represents a Reynolds number of the turbulence inside the entrainment zone.

Statistical properties depend on the set of variables $\{z, t\}$, which can be nondimensionalized as $\left\{z / L_{0}, t N\right\}$. However, without loss of generality, we substitute them by the equivalent set of independent variables $\left\{z / z_{\text {enc }}\right.$, $\left.z_{\text {end }} / L_{0}\right\}$, where

$$
z_{\text {enc }}(t)=\left[\frac{2 B_{0}}{N^{2}}\left(1+\mathrm{Re}_{0}^{-1}\right)\left(t-t_{0}\right)\right]^{1 / 2}
$$

is the encroachment height (Lilly 1968; Carson and Smith 1975). The virtual time origin $t_{0}$ quantifies the dependence on the initial condition $\langle b\rangle(z, 0)$ and is defined such that 
$\left(1+\mathrm{Re}_{0}^{-1}\right) B_{0}\left(t-t_{0}\right)=\int_{0}^{z_{\infty}}\left[\langle b\rangle(z, t)-b_{0}(z)\right] d z$

for any given time $t$, where $b_{0}(z)$ is the reference background buoyancy profile, Eq. (2), and $z_{\infty}$ is located far enough into the nonturbulent stably stratified region. Angle brackets denote averaging along horizontal planes hereinafter.

Since $z_{\mathrm{enc}}$ is commensurate with the CBL thickness for the case of a CBL growing into a linearly stratified atmosphere, the independent variable $z / z_{\text {enc }}$ (or equivalent normalized height) is often used to study the self-similar behavior of the CBL as it grows in time. In contrast, the use of

$$
z_{\text {enc }} / L_{0}=\left[2\left(1+\operatorname{Re}_{0}^{-1}\right)\left(t-t_{0}\right) N\right]^{1 / 2}
$$

instead of $t N$ to measure the state of development of the CBL is less common. The reason to use it in this work is twofold. First, according to the interpretation of $L_{0}$ introduced before and further discussed in section $5, z_{\text {end }} / L_{0}$ is a measure of the ratio between the integral length scale of turbulence within the mixed layer and the integral length scale within the entrainment zone and is therefore a relevant measure of scale separation in the CBL. Second, $z_{\text {end }} / L_{0}$ retains the effect of both $N$ and $B_{0}$ simultaneously, meaning that both the weak stratification regime (relatively thick EZ) and the strong stratification regime (relatively thin EZ) can be covered by a single simulation as $z_{\text {end }} / L_{0}$ grows in time. According to the results presented in the following section, the distinction between the weak and strong stratification regimes occurs at $z_{\text {end }} / L_{0} \simeq 10$.

For comparison with the atmospheric CBL, we use the estimates $L_{0} \simeq 20-200 \mathrm{~m}$ [derived from typical values $N \simeq 0.6-1.8 \times 10^{-2} \mathrm{~s}^{-1}$ and $B_{0} \simeq 0.3-1.0 \times 10^{-2} \mathrm{~m}^{2} \mathrm{~s}^{-3}$ (Fedorovich et al. 2004a; Träumner et al. 2011)], $z_{\text {enc }} \simeq$ $1000 \mathrm{~m}$, and $\nu=1.5 \times 10^{-5} \mathrm{~m}^{2} \mathrm{~s}^{-1}$ to obtain $z_{\text {end }} / L_{0} \simeq 5$ 50 and $\mathrm{Re}_{0} \simeq 6 \times 10^{5}$ to $2 \times 10^{7}$. Our simulations reach up to $z_{\text {end }} / L_{0} \simeq 26$ (see Table 1 ) and therefore the range of values achieved is representative of atmospheric conditions and encompasses the different stratification regimes considered in Fedorovich et al. (2004a), whose weakest and strongest stratification cases correspond to values $z_{\text {end }} / L_{0} \simeq 7.3$ and $z_{\text {end }} / L_{0} \simeq 23.5$, respectively. On the other hand, the Reynolds numbers $\operatorname{Re}_{0}=42$ and $\mathrm{Re}_{0}=117$ in our simulations are still orders of magnitude smaller than atmospheric values. However, by comparing both cases, we show throughout the paper (see also appendix B) that Reynolds number effects on the results that we discuss are negligibly small.

Notice that the reference Reynolds number $\operatorname{Re}_{0}$ characterizing the turbulence inside the $\mathrm{EZ}$ is different from, but related to, the convective Reynolds number

$$
\mathrm{Re}_{*}=\frac{z_{\mathrm{enc} w_{*}}}{\nu}=\left(\frac{z_{\mathrm{enc}}}{L_{0}}\right)^{4 / 3} \operatorname{Re}_{0}
$$

that is associated with the large-scale convective motions and that characterizes the turbulence inside the mixed layer, where

$$
w_{*}=\left(B_{0} z_{\mathrm{enc}}\right)^{1 / 3}
$$

is the convective velocity (Deardorff 1970). As shown later on, some of the large-scale statistics in the CBL begin to show a relatively small dependence on the Reynolds number as the simulations approach values $\mathrm{Re}_{*} \simeq 0.5 \times 10^{4}$ (Table 1 ), which is in agreement with the behavior found in other turbulent flows for outerscale Reynolds numbers beyond $O\left(10^{4}\right)$, as discussed by Dimotakis (2000).

For reference, the range of $\mathrm{Re}_{*}$ values achieved in simulation $\mathrm{Re} 040$ covers the values achieved in the experiments of Deardorff et al. (1980) in a $1.11 \mathrm{~m} \times 1.24 \mathrm{~m}$ wide water tank. However, those tank experiments have quite a low $\mathrm{Re}_{0}$, about 10; they achieve a comparable $\mathrm{Re}_{*}$ because $z_{\text {end }} / L_{0}$ is larger, about 60 . (To reach an $\mathrm{Re}_{*}$ similar to that of simulation Re100, the CBL of the tank experiment would have to grow from 0.27 to $0.50 \mathrm{~m}$, and to obtain a similar aspect ratio of the horizontal dimension to the CBL thickness as in the simulations, the tank would need to be $6 \mathrm{~m} \times 6 \mathrm{~m}$ wide.)

\section{Preliminaries}

We briefly discuss in this section two topics that are relevant for the following sections. First, we evaluate different definitions of the height of the CBL top because they provide reference positions needed for the detailed analysis of the entrainment zone presented in section 5 . Second, we show that beyond $z_{\text {end }} / L_{0} \simeq 10$, the CBL is already within the equilibrium entrainment regime.

\section{a. Definitions of the height of the convective boundary layer top}

We consider the following definitions of the height of the CBL top, $z_{i, \xi}$, as introduced before in the literature (see, e.g., Garratt 1992; Sullivan et al. 1998):

(i) The zero-crossing height $z_{i, 0}$, where the total buoyancy flux

$$
B=\left\langle b^{\prime} w^{\prime}\right\rangle-\kappa \frac{\partial\langle b\rangle}{\partial z}
$$

becomes negative. A prime indicates turbulent fluctuation (deviation from the horizontal plane average). 


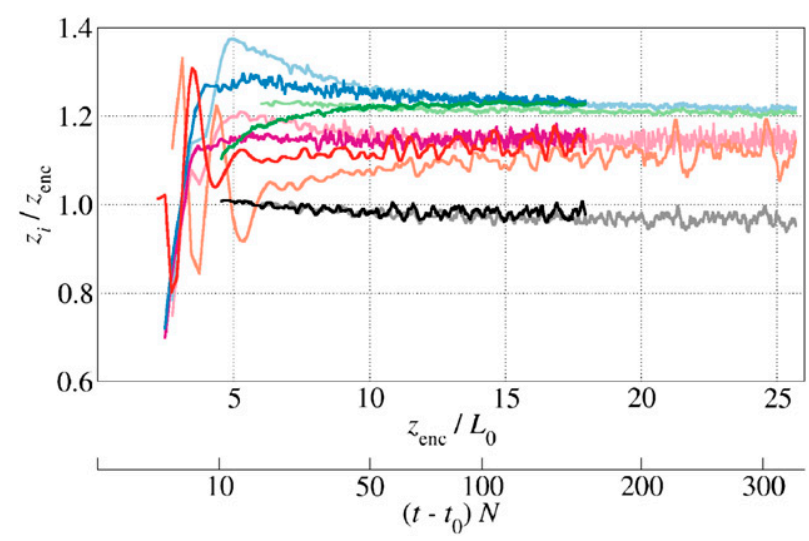

FIG. 1. Temporal evolution of the normalized CBL-top heights, defined in section 4a: zero crossing of buoyancy flux $z_{i, 0}$ (black), integral-based $z_{i, i}$ (red), flux-based $z_{i, f}$ (magenta), variance-based $z_{i, v}$ (green), and gradient-based $z_{i, g}$ (blue). The corresponding mean values beyond $z_{\text {end }} / L_{0} \simeq 10$ are summarized in Table 2 . Light colors correspond to Re040, dark colors correspond to Re100.

(ii) The integral-based height

$$
z_{i, i}(t)=2.5 \frac{1}{B_{0}} \int_{0}^{z_{\infty}}\left\langle b^{\prime} w^{\prime}\right\rangle d z
$$

where the integral extends far enough into the nonturbulent stably stratified region. The factor 2.5 comes from assuming a linearly decreasing buoyancy flux profile that has a minimum buoyancy flux value of $-0.2 B_{0}$ (see Deardorff 1970).

(iii) The flux-based height $z_{i, f}$, where the total buoyancy flux $B$ is minimum.

(iv) The variance-based height $z_{i, v}$, where the buoyancy variance $\left\langle b^{\prime} b^{\prime}\right\rangle$ is maximum away from the nearwall region.

(v) The gradient-based height $z_{i, g}$, where the mean buoyancy gradient is maximum away from the near-wall region.

We compare these heights, normalized with the encroachment height $z_{\text {enc }}$ [Eq. (5)], in Fig. 1. When the thickness of the CBL becomes an order of magnitude larger than $L_{0}$, all of the normalized heights become statistically steady in time, within the statistical convergence that we can achieve. Table 2 summarizes the values of the corresponding constant

$$
C_{\mathrm{enc}, \xi}=z_{i, \xi} / z_{\mathrm{enc}} \text {. }
$$

This steadiness implies that $z_{\text {enc }}$ already gives the correct evolution in time of the growth of the dry CBL into a linearly stratified fluid (Driedonks 1982). The mean entrainment rate

$$
w_{e, \xi}=d z_{i, \xi} / d t
$$

TABLE 2. Normalized CBL-top height constants $C_{\text {enc, },}$, Eq. (12), calculated for all the different height definitions $\left\{z_{i, \xi}: \xi=0, i, f, v, g\right\}$ introduced in section $4 \mathrm{a}$ and shown in Fig. 1, using the data for $z_{\text {end }}$ ' $L_{0} \geq 10$.

\begin{tabular}{lllllll}
\hline \hline & Heights & $z_{i, 0}$ & $z_{i, i}$ & $z_{i, f}$ & $z_{i, v}$ & $z_{i, g}$ \\
\hline Re040 & Mean & 0.97 & 1.11 & 1.15 & 1.21 & 1.23 \\
& $\sigma(\%)$ & 1.0 & 2.1 & 1.1 & 0.36 & 0.83 \\
Re100 & Mean & 0.98 & 1.13 & 1.15 & 1.23 & 1.24 \\
& $\sigma(\%)$ & 1.0 & 1.6 & 0.9 & 0.29 & 0.54 \\
\hline
\end{tabular}

can then be approximated as

$$
w_{e, \xi} \simeq C_{\mathrm{enc}, \xi} \frac{d z_{\mathrm{enc}}}{d t}=C_{\mathrm{enc}, \xi} N L_{0}\left(z_{\mathrm{enc}} / L_{0}\right)^{-1}
$$

for $z_{\mathrm{end}} / L_{0} \geq 10$. Hence, the range of values of $C_{\mathrm{enc}, \xi}$ gives a variability of $\simeq 25 \%$ in $w_{e}$ depending on the height $z_{i, \xi}$ that is used to define the CBL top. In particular, the mean entrainment rate differs by roughly $10 \%$ between the common CBL-top height definitions $z_{i, f}$ and $z_{i, g}$. We will denote $d z_{\text {enc }} / d t$ as $w_{e}$.

We emphasize that, according to Fig. 1 and Table 2, the Reynolds number dependence of the entrainment rate $w_{e}$ after the initial transient, beyond $z_{\text {end }} / L_{0} \simeq 5-$ 10 , is already negligibly small for the Reynolds numbers achieved in these simulations, only about $2 \%$ and comparable to the statistical convergence.

The different CBL-top heights are ordered according to

$$
z_{i, 0} \leq z_{\mathrm{enc}}<z_{i, i} \leq z_{i, f}<z_{i, v} \leq z_{i, g}
$$

in agreement with previous results (Sullivan et al. 1998; Fedorovich et al. 2004a). Approximately, there are only three distinct heights since we observe that $z_{i, 0} \simeq z_{\text {enc }}$, $z_{i, i} \simeq z_{i, f}$, and $z_{i, v} \simeq z_{i, g}$. These three heights are depicted as three white bars in Fig. 2, which visualizes a vertical cross section of the CBL using the magnitude of the buoyancy gradient. The entrainment zone, whose location is roughly indicated by these three heights, is dominated by both sharp gradients at the crests of the undulations, or domes, and the entrained fluid regions between those domes, or troughs. The smallest of the three heights, the zero-crossing height of the buoyancy flux, was used in the original study of Deardorff et al. (1980), and is said to characterize the top of the wellmixed layer, which is supported by the visualization in Fig. 2 (see also Fig. 6d). The largest of those heights, $z_{i, g}$, seems to mark the mean vertical extent reached by the penetrating thermals. We will discuss in section 5 and section 6 the details of this entrainment region that develops between $z_{i, 0}$ and $z_{i, g}$. 


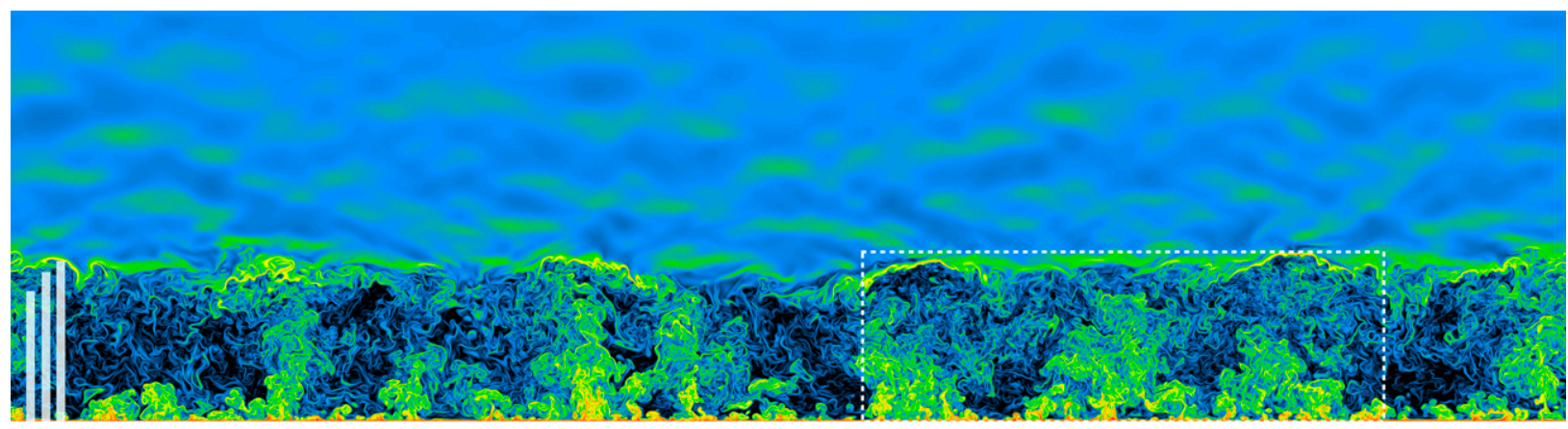

FIG. 2. Vertical cross section of the CBL showing the logarithms of the magnitude of the buoyancy gradient $N^{-2}|\nabla b|$ for case Re100 at the final time $z_{\text {enc }} / L_{0}=18$. Colors black, blue, yellow, and red correspond, respectively, to values varying between $10^{-1}$ and $10^{2}$ in powers of 10. Color scale is as Fig. 6. The tops of the three white vertical bars indicate the location of the different CBL height definitions, from left to right: the encroachment height $z_{\text {enc }}$, the flux-based height $z_{i, f}$, and the gradient-based height $z_{i, g}$.

\section{b. The equilibrium entrainment regime}

To show that we are analyzing statistics within the equilibrium entrainment regime, we follow Fedorovich et al. (2004a) and perform an integral analysis of the evolution equation for the turbulence kinetic energy

$$
\frac{\partial e}{\partial t}+\frac{\partial T}{\partial z}=\left\langle b^{\prime} w^{\prime}\right\rangle-\varepsilon
$$

where $T=\left\langle w^{\prime} v_{i}^{\prime} v_{i}^{\prime} / 2+p^{\prime} w^{\prime}-u_{i}^{\prime} \tau_{i z}^{\prime}\right\rangle$ is the turbulent flux of kinetic energy in the vertical direction, and $\varepsilon=\left\langle u_{i, j}^{\prime} \tau_{i, j}^{\prime}\right\rangle$ is the mean viscous dissipation rate, with the viscous stress tensor being $\tau_{i j}=\nu\left(u_{i, j}+u_{j, i}\right)$ (the symbol $u_{i, j}$ denotes partial derivative in the direction $j$ for the velocity component $u_{i}$ ). Integrating this transport equation from the surface up to a height $z_{\infty}$ located far enough into the nonturbulent stably stratified region yields

$$
C_{t}+C_{T}=C_{b w}-C_{\varepsilon},
$$

where $C_{t}=w_{*, i}^{-3} \int_{0}^{z_{\infty}}(\partial e / \partial t) d z, C_{T}=w_{*, i}^{-3} T\left(z_{\infty}\right)$, and the terms at the right-hand side are

$$
C_{b w}=w_{*, i}^{-3} \int_{0}^{z_{\infty}}\left\langle b^{\prime} w^{\prime}\right\rangle d z, \quad C_{\varepsilon}=w_{*, i}^{-3} \int_{0}^{z_{\infty}} \varepsilon d z
$$

The convective velocity in the expressions above is $w_{*, i}=$ $\left(B_{0} z_{i, i}\right)^{1 / 3}$ (Deardorff 1970). By construction, $C_{b w}=0.4$.

The evolution in time of the terms in Eq. (17), shown in Fig. 3a, has three main features. First, the collapse of the curves from simulations Re040 and Re100, particularly that of $C_{\varepsilon}$, indicates the tendency of the production and destruction rates of turbulence kinetic energy toward an inviscid scaling that depends solely on the integral scales $z_{i, i}$ and $w_{*, i}$ (i.e., independent of the viscosity). This behavior is another manifestation of Reynolds number similarity (Tennekes and Lumley 1972; Pope 2000; Monin and Yaglom 2007). Second, the negligibly small transport term, $C_{T}$, implies a negligible energy drain due to the upward radiation of inertial gravity waves, in accord with previous findings in similar CBL configurations (Deardorff et al. 1980; Carruthers and Hunt 1986; Fedorovich et al. 2004a). Finally, the decreasing tendency term $C_{t}$ implies that beyond $z_{\text {end }}$ l $L_{0} \simeq 10$, the dominant balance in Eq. (17) is between the terms $C_{b w}$ and $C_{\varepsilon}$, meaning that

$$
C_{b w} \simeq C_{\varepsilon} .
$$

This balance corresponds to the equilibrium entrainment regime (Fedorovich et al. 2004a). Within this regime, the $\mathrm{CBL}$ is in a quasi-steady state in the sense that the time $z_{\text {end }} / w_{e}=N^{-1}\left(z_{\text {end }} / L_{0}\right)^{2}$ required for a significant change of the CBL thickness is much longer than the turnover time $z_{\text {end }} / w_{*}=N^{-1}\left(z_{\text {enc }} / L_{0}\right)^{2 / 3}$ associated with the large-scale convective motions inside the CBL.
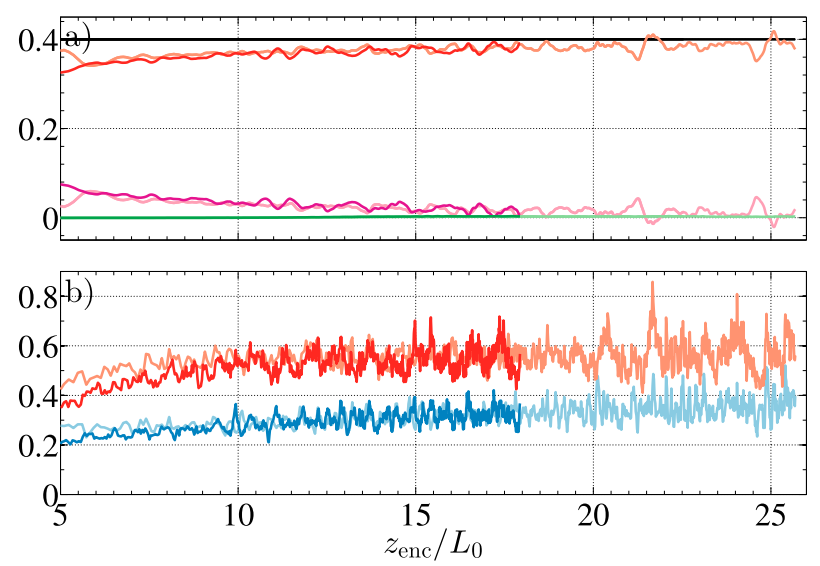

FIG. 3. (a) Temporal evolution of normalized terms of the integral budget equation of turbulence kinetic energy, Eq. (17), showing the dominant balance $C_{b w} \simeq C_{\varepsilon}: C_{\varepsilon}$ (red), $C_{t}$ (magenta), and $C_{T}$ (green); $C_{b w}=0.4$ (black), by definition. (b) Temporal evolution of the normalized terms of the local budget equation of turbulence kinetic energy, Eq. (16), $\varepsilon /(-\partial T / \partial z)$ (red) and $-\left\langle b^{\prime} w^{\prime}\right\rangle /(-\partial T / \partial z)$ (blue), evaluated at the height of minimum buoyancy flux $z_{i, f}$. Light colors correspond to Re 040 , dark colors correspond to Re100. 

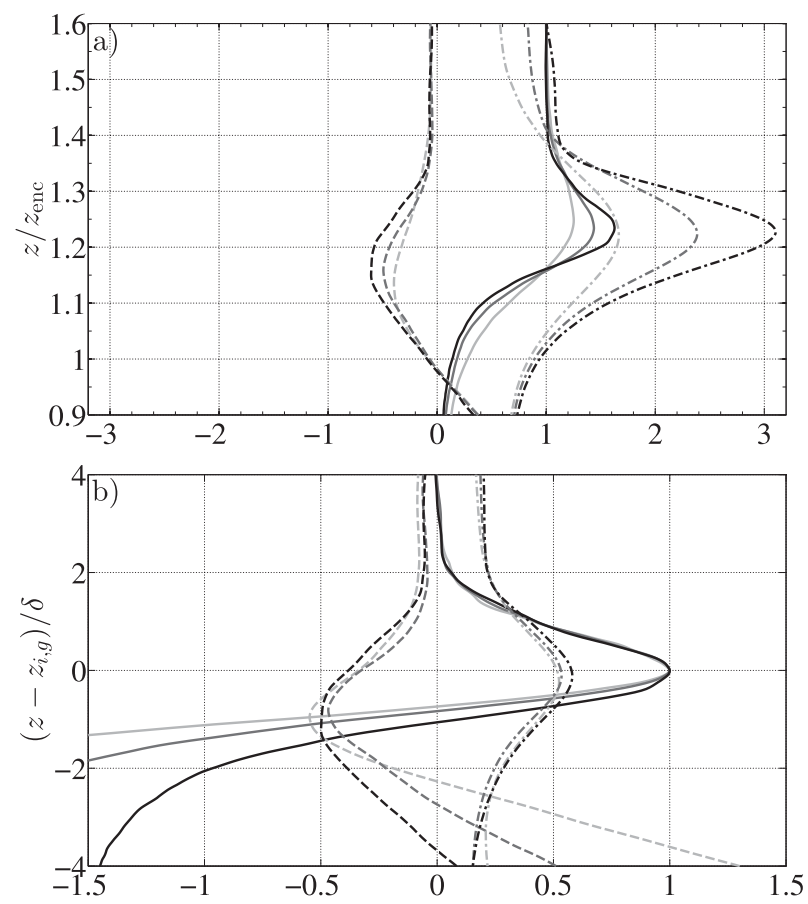

FIG. 4. (a) Vertical profiles inside the entrainment zone normalized by $z_{\text {enc }}:(\partial\langle b\rangle / \partial z) / N^{2}$ (solid), $b_{\text {rms }} / b_{*}$ (dash dotted), and $B /$ $B_{0}$ multiplied by a factor of 5 for emphasis (dashed). (b) Vertical profiles inside the entrainment zone centered at $z_{i, g}$ and normalized by $\delta:\left(\partial\langle b\rangle / \partial z-N^{2}\right) /\left(b_{\delta} / \delta-N^{2}\right)$ (solid), $b_{\text {rms }} / b_{\delta}$ (dash-dotted), and $B /\left[b_{\delta} w_{\text {rms }}\left(z_{i, \mathrm{~g}}\right)\right]$ multiplied by a factor of 5 for emphasis (dashed). Light gray, gray, and black indicate snapshots from simulation $\operatorname{Re} 100$ at $z_{\text {enc }} / L_{0} \simeq\{10,14,18\}$, respectively.

Indeed, the ratio of these two time scales, $w_{*} / w_{e}=$ $\left(z_{\text {end }} / L_{0}\right)^{4 / 3}$, is larger than an order of magnitude for the interval of the normalized CBL thickness $z_{\text {enc }} / L_{0} \geq 10$ considered in this work.

\section{Vertical structure of the entrainment zone}

In previous studies of the CBL vertical structure, the entrainment zone was often considered as a single layer at the top of the well-mixed region (see, e.g., Deardorff et al. 1980; Sullivan et al. 1998; Fedorovich et al. 2004a, and references therein). In contrast, we find that the entrainment zone is better described as a composition of two overlapping layers, based on the observation that local properties, like length and buoyancy scales, evolve differently in time within each of those two layers. We clarify this as follows.

On the one hand, the proportionality among the different definitions of the CBL-top height discussed in the previous section (Fig. 1) indicates that we can define a first sublayer in the entrainment zone with a thickness $z_{i, g}-z_{i, 0}$ that is proportional to the CBL thickness. Using the coefficients from Table 2, we can write $z_{i, g}-$ $z_{i, 0}=\left(C_{\mathrm{enc}, g}-C_{\mathrm{enc}, 0}\right) z_{\mathrm{enc}} \simeq 0.25 z_{\mathrm{enc}}$.

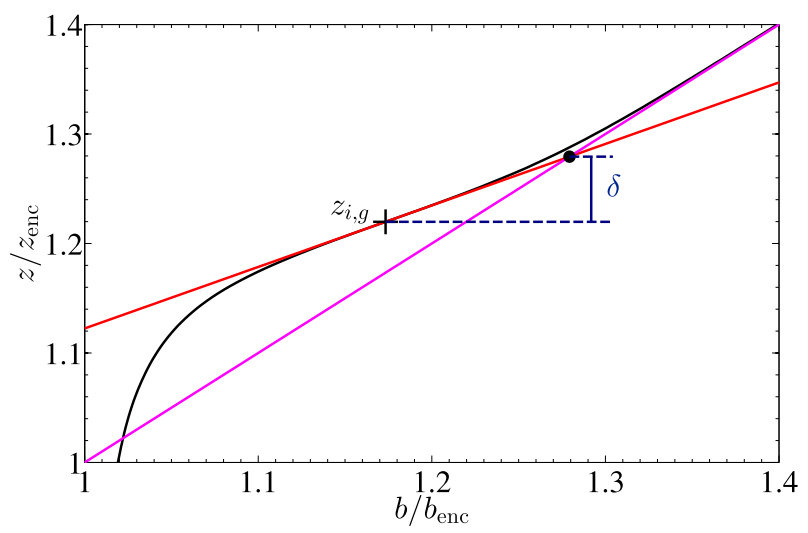

FIG. 5. Sketch illustrating the definition of the local thickness $\delta$, Eq. (21), characterizing the upper EZ sublayer. The mean buoyancy profile (solid black) corresponds to the final time of simulation $\operatorname{Re} 040\left(z_{\text {enc }} / L_{0} \simeq 26\right)$; tangent line at the point of maximum buoyancy gradient (marked with a plus sign) is shown in red; the background buoyancy profile is shown in magenta.

On the other hand, as we approach the height of maximum mean buoyancy gradient $z_{i, g}$, the vertical profiles of the mean buoyancy gradient and of the root-mean-square (rms) of the buoyancy fluctuations, $b_{\mathrm{rms}}=\left\langle b^{\prime} b^{\prime}\right\rangle^{1 / 2}$, do not scale with either the encroachment scales or the convective scales; as shown in Fig. 4a, around the height $z_{i, g}$, the magnitudes of these normalized quantities keep increasing in time. This behavior suggests that we consider an upper EZ sublayer centered at $z_{i, g}$ whose thickness is not a constant fraction of the CBL thickness, but instead is proportional to a local length scale. One possible definition of this characteristic length scale is based on the relation

$$
\langle b\rangle\left(z_{i, g}\right)+\frac{\partial\langle b\rangle}{\partial z}\left(z_{i, g}\right) \delta=b_{0}\left(z_{i, g}\right)+N^{2} \delta,
$$

sketched in Fig. 5. This expression can be written as

$$
\delta=-\frac{\langle b\rangle\left(z_{i, g}\right)-b_{0}\left(z_{i, g}\right)}{\frac{\partial\langle b\rangle}{\partial z}\left(z_{i, g}\right)-N^{2}} .
$$

This is a gradient thickness definition that is often employed in the characterization of turbulent mixing layers that separate two regions where the flow is statistically homogeneous in the two directions perpendicular to the mean gradient (see, e.g., Pope 2000). Hence, the definition (21) supports the interpretation of the upper EZ sublayer as a transition region, based on the buoyancy field, between the nonturbulent, stably stratified fluid above and the turbulent layer below. The corresponding buoyancy scale within the upper EZ sublayer is

$$
b_{\delta}=\frac{\partial\langle b\rangle}{\partial z}\left(z_{i, g}\right) \delta
$$


which is similar to the scaling proposed by Sorbjan (1999).

When normalized with $\delta$ and $b_{\delta}$, the profiles of mean buoyancy gradient and buoyancy rms at different times approximately collapse on top of each other (Fig. 4b). This self-similar behavior of the buoyancy profiles resembles the self-similarity hypothesis that underlies the general structure models of the EZ (Fedorovich and Mironov 1995; Fedorovich et al. 2004a), although we find that it applies only within the upper EZ sublayer, namely, in a region $z_{i, g} \pm \delta$, and not across the entire entrainment zone, as originally postulated in those models.

For the rest of this section, we discuss the physical mechanism that causes this mixing region $z_{i, g} \pm \delta$ and the interpretation of the characteristic scales $\delta$ and $b_{\delta}$. In addition, we also provide explicit parameterizations for these scales as a function of the independent variable $z_{\text {end }} / L_{0}$, so that the buoyancy profiles inside the EZ can be reconstructed at any time, if desired.

\section{a. The length scale}

\section{1) MEAN PENETRATION DEPTH OF THERMALS}

Figure 2 and, with more detail, Figs. 6a and 6b illustrate that the upper EZ sublayer is a region characterized by turbulent thermals penetrating into a smoothly varying environment. Qualitatively, we can interpret the height $z_{i, g}$ and the thickness $\delta$, respectively, as a rough estimate of the mean and standard deviation of the vertical location of the top of the interface undulations, or domes, that are created by the overshooting thermals (Fig. 7). On the other hand, the lower EZ sublayer is characterized by the updrafts, acting as pillars that support the domes in the upper EZ sublayer, and by the turbulent troughs in between the domes (Figs. 6c,d). Within this lower EZ sublayer, we find strong fluctuations in the buoyancy field all across the horizontal extent.

The interpretation of $\delta$ as the mean penetration depth above $z_{i, g}$ that the thermals reach is supported by parcel theory. This theory states that given a parcel of fluid with a vertical velocity $w^{\prime}$ at its neutral buoyancy level, the vertical displacement reached by this parcel of fluid inside a linearly stratified region with buoyancy frequency $N$ is proportional to $w^{\prime} / N$ (Zeman and Tennekes 1977; Xuequan and Hopfinger 1986; Hopfinger 1987; Smyth and Moum 2000).

For the fluid parcels at $z_{i, g}$, we can propose that $w^{\prime} \sim$ $w_{\text {rms }}\left(z_{i, g}\right)$. At the same time, we observe in our simulation that the integral velocity scale of the turbulence inside the upper EZ sublayer is a constant fraction of the convective velocity,

$$
w_{\mathrm{rms}}\left(z_{i, g}\right) \simeq c_{w 2} w_{*}
$$

(Fig. 8a), beyond $z_{\text {end }} / L_{0} \simeq 10$, where $c_{w 2} \simeq 0.2$ (Table 3 ). Therefore, we can write

$$
\delta \simeq c_{\delta}\left(w_{*} / N\right) .
$$

Indeed, Fig. $8 \mathrm{~b}$ demonstrates that $\delta$ follows this scaling within the equilibrium entrainment regime, beyond $z_{\text {end }} / L_{0} \simeq 10$, and that $c_{\delta} \simeq 0.55$. The Reynolds number dependence of this constant is already negligibly small for the Reynolds numbers $\operatorname{Re}_{0} \simeq 100$ achieved in our simulations (about $2 \%$, less than the uncertainty $\simeq 5 \%$ associated with the statistical convergence; see Table 3 ).

From Eq. (24) and the definition of $w_{*}$, we see that $\delta$ is actually increasing in time according to

$$
\delta / L_{0}=c_{\delta}\left(z_{\text {enc }} / L_{0}\right)^{1 / 3},
$$

but, with respect to $z_{\mathrm{enc}}$, it continuously decreases as

$$
\delta / z_{\text {enc }}=c_{\delta}\left(z_{\text {enc }} / L_{0}\right)^{-2 / 3} .
$$

This scaling of $\delta$ highlights the effect of the stratification on the geometry of the turbulent structures inside the entrainment zone. If there were no stratification $\left(N^{2}=0\right)$, the size of the undulations due to the thermals would scale with the boundary layer thickness and entrainment would be dominated by large-scale engulfment (Mellado 2012). With stratification, an interval $z_{i, g}-z_{i, 0}$ of the entrainment zone retains the scaling proportional to the CBL thickness $z_{\text {enc }}$, but a second sublayer develops within the region of the EZ that is closer to the stably stratified layer. This upper EZ sublayer can be interpreted as a transition region between the convectively mixed layer, characterized to leading order by $z_{\text {enc }}$ and $w_{*}$, and the stably stratified layer above, characterized by $w_{*}$ and $N$. The properties of this upper EZ sublayer depend directly on $N$, in contrast to those inside the lower EZ sublayer, where $N$ enters only indirectly through the dependence of the CBL thickness $z_{\text {enc }}$ on the buoyancy frequency $N$ as in Eq. (5).

Finally, the scaling above also means that, as the CBL grows, the upper EZ sublayer, with extent $z_{i, g} \pm \delta$, becomes a smaller fraction of the lower EZ sublayer, with an extent $\left(z_{i, g}-z_{i, 0}\right) \simeq 0.25 z_{\text {enc. }}$ Notice, however, that for a significant range of typical atmospheric conditions $\left(z_{\text {enc }} / L_{0} \simeq 10-50\right.$; see section 3$)$, the upper EZ sublayer still occupies a significant fraction of the entrainment zone, since $2 \delta /\left(z_{i, g}-z_{i, 0}\right)$ varies between 0.95 and 0.46 within this range.

\section{2) INTEGRAL LENGTH SCALE OF TURBULENCE}

The existence of a turbulence integral length inside the EZ that is different from the CBL thickness $z_{\text {enc }}$ (or a constant fraction thereof) has previously been 
(a)

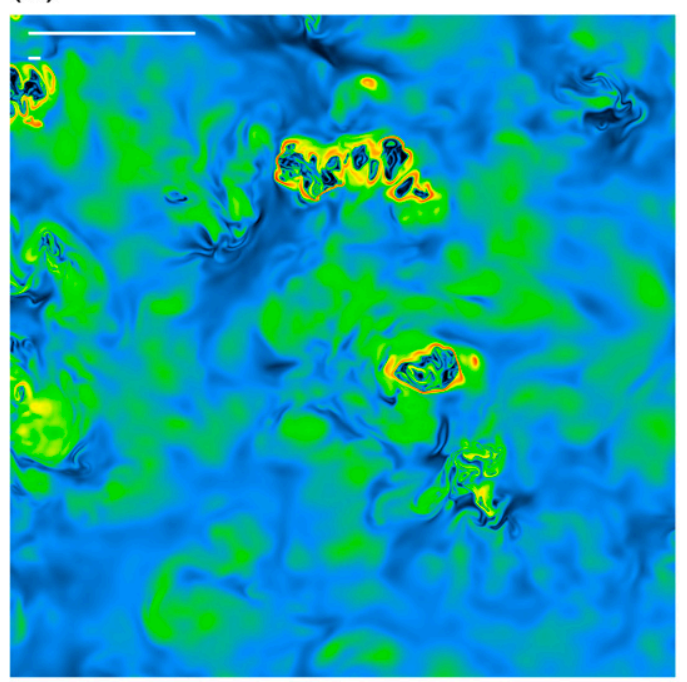

(c)

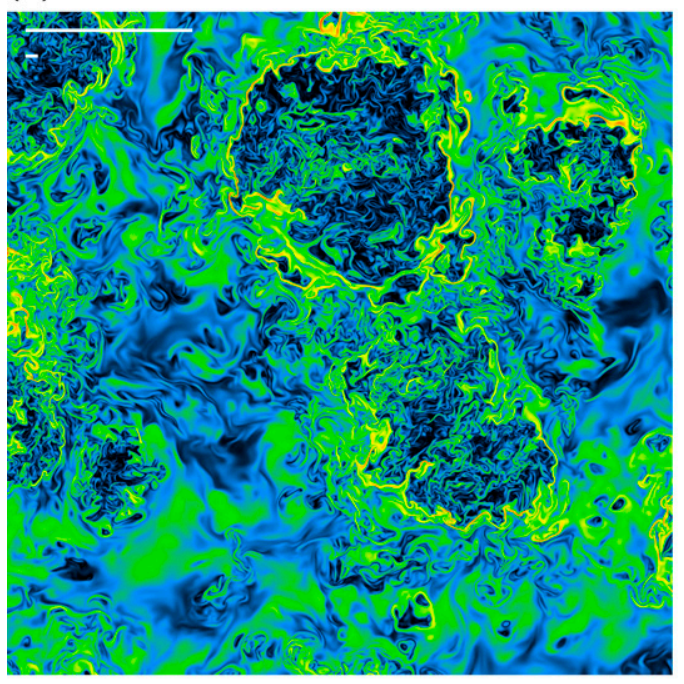

(b)

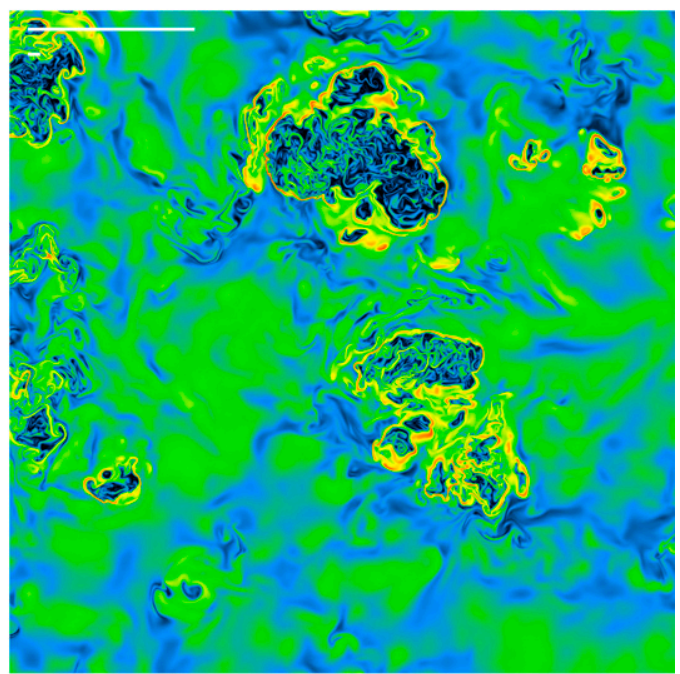

(d)

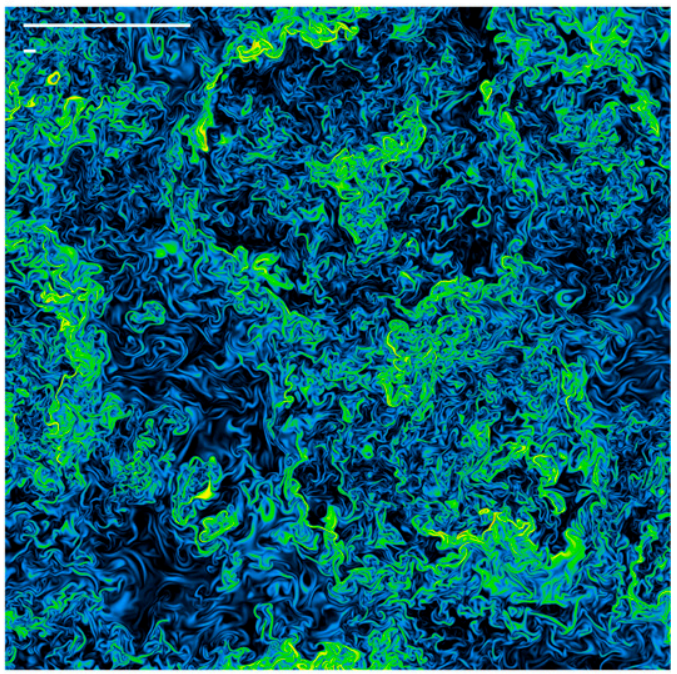

\section{$\log _{10}\left(N^{-2}|\nabla \mathrm{b}|\right)$}

FIG. 6. Horizontal cross sections showing the logarithm of the magnitude of the buoyancy gradient $N^{-2}|\nabla b|$ inside the entrainment zone for the case Re100 at the final time $z_{\text {enc }} / L_{0}=18$ (only $1 / 9$ of the domain is shown). Colors black, blue, yellow, and red correspond, respectively, to values varying between $10^{-1}$ and $10^{2}$ in powers of 10 . The heights are (a) $z=z_{i, 1}$, (b) $z=z_{i, g}$, (c) $z=z_{i, f}$, and (d) $z=z_{i, 0}$. The long horizontal white bar at the top left corner of each panel indicates a length equal to $z_{\text {enc }}$, Eq. (5); the short white bar indicates a length equal to $\delta$, Eq. (21).

considered due to increasing evidence that the viscous dissipation rate $\varepsilon$ inside the $\mathrm{EZ}$ is not a fixed fraction of the rate of energy input into the system (Linden 1975; Tennekes 1975; Guillemet et al. 1983). The reason for this behavior was attributed to the influence of stratification on the turbulence near the stratified interface (Zeman and Tennekes 1977; Mahrt 1979). The scaling

$$
\varepsilon\left(z_{i, g}, t\right) \simeq c_{\varepsilon}\left[w_{\text {rms }}\left(z_{i, g}\right)\right]^{3} / \delta
$$

observed in Fig. $8 \mathrm{c}$ for roughly $z_{\text {end }} / L_{0} \geq 10-15$, where $c_{\varepsilon} \simeq 0.5$, supports those hypotheses. In particular, this inviscid scaling of the viscous dissipation rate implies that $\delta$ is not only the mean penetration depth of thermals, but it also represents the integral length scale of the turbulence inside the crests or domes of the overshooting thermals (Pope 2000).

Consistent with the scalings Eqs. (24) and (27), the Ozmidov scale within these turbulence regions or 


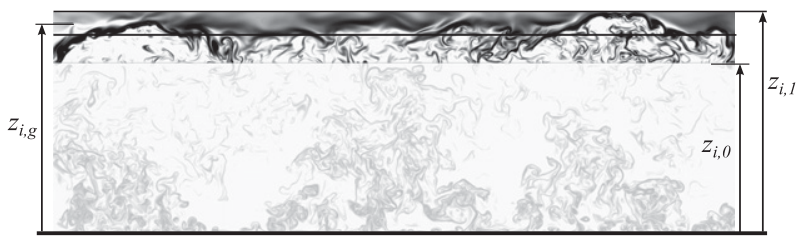

FIG. 7. Extracted region from Fig. 2 (see dashed white box) emphasizing the entrainment zone. The upper EZ sublayer, $z_{i, g} \pm \delta$ (enclosed by two horizontal lines, where $z_{i, 1}=z_{i, g}+\delta$ ), features the overshooting thermals, and corresponds to the region directly affected by the stably stratified overlying fluid. The lower EZ sublayer, $z_{i, g}-z_{i, 0}$, features the troughs of the undulations there. The layer below $z_{i, 0}$ (masked region) is the well-mixed layer.

turbulence pockets inside the upper EZ sublayer (Figs. $6 \mathrm{a}, \mathrm{b})$ is proportional to $\delta$ according to

$$
\left[\frac{\varepsilon\left(z_{i, g}, t\right)}{N^{3}}\right]^{1 / 2}=\left(c_{\varepsilon} c_{w 2}^{3} / c_{\delta}^{3}\right)^{1 / 2} \delta,
$$

with $\left(c_{\varepsilon} c_{w 2}^{3} / c_{\delta}^{3}\right)^{1 / 2} \simeq 0.2$ (Table 3 ). The Ozmidov scale characterizes the size of the largest eddies in fully developed turbulence under a homogeneous stratification (Ozmidov 1965; Smyth and Moum 2000) and thus the local integral scale, $\delta$ in our case. Interestingly, the reference Ozmidov scale $L_{0}$ is actually comparable in magnitude to $\delta$ within the interval $z_{\text {end }} / L_{0} \simeq 10-26$ (see Table 1). Therefore, $L_{0}$ provides a first estimate of the characteristic length $\delta$ for this range of typical atmospheric conditions. This also implies that the reference Reynolds number $\mathrm{Re}_{0}$ is not only a control parameter of the problem, but also approximates well the Reynolds number $\operatorname{Re}_{\delta}$ that characterizes the turbulence inside part of the entrainment zone, since

$$
\operatorname{Re}_{\delta}=\frac{\delta w_{\mathrm{rms}}\left(z_{i, g}\right)}{\nu}=\left(c_{w 2} / c_{\delta}\right) \operatorname{Re}_{0}\left(\delta / L_{0}\right)^{2},
$$

where $c_{w 2} / c_{\delta} \simeq 0.44$ (Table 3 ) and $\delta / L_{0}$ varies between 1.1 and 1.9 [Eq. (25)] for $z_{\text {end }} / L_{0}$ varying between 10 and 50 .

In sum, our results confirm the previous hypothesis (Zeman and Tennekes 1977; Mahrt 1979) that the integral length scale of the turbulence locally within the entrainment zone is modified by the stable stratification, more specifically, within the upper EZ sublayer. In addition, we have provided an explicit parameterization of this integral length scale in terms of the independent variable $z_{\text {end }} / L_{0}$ in Eq. (25).

\section{b. The buoyancy scale}

From Eqs. (21) and (22), the buoyancy scale $b_{\delta}$ can be equivalently defined as
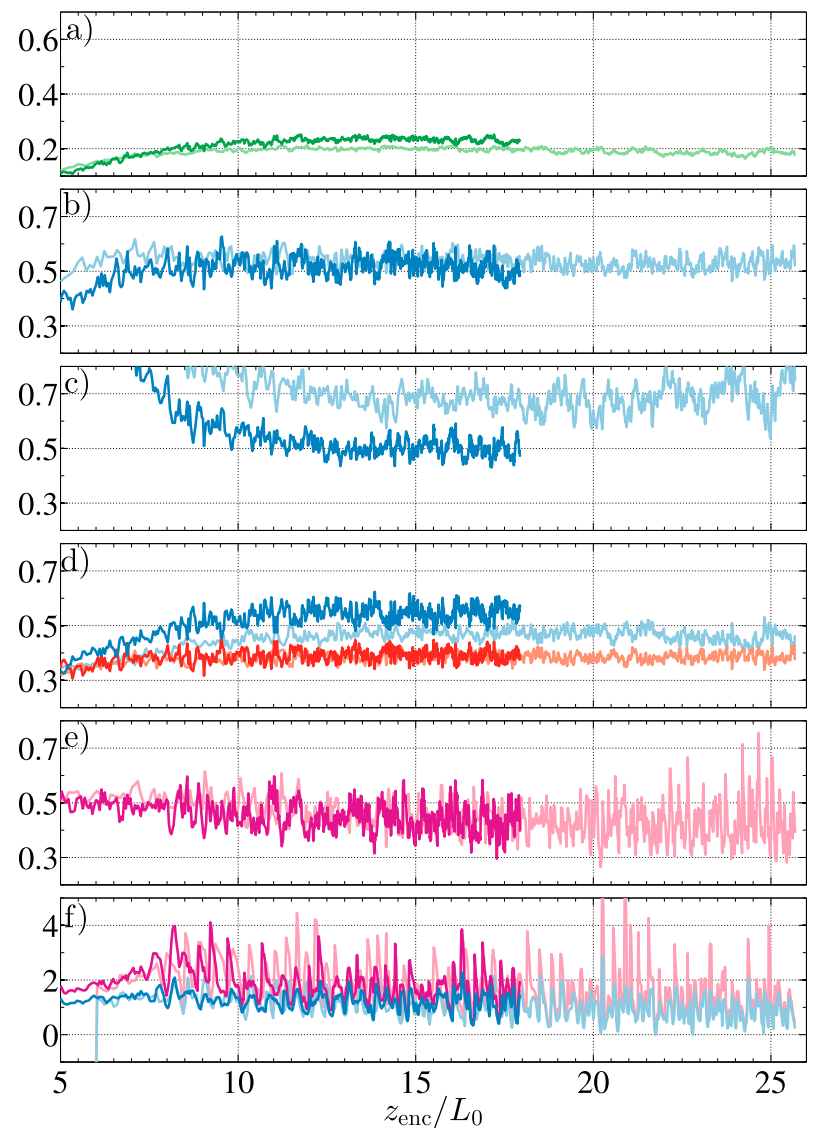

FIG. 8. Temporal evolution of the characteristic scales inside the upper EZ sublayer: (a) velocity, $w_{\text {rms }}\left(z_{i, g}\right) / w_{*} ;$ (b) length, $\delta /\left(w_{*} / N\right)$; (c) local inviscid scaling of the viscous dissipation rate, $\delta \varepsilon\left(z_{i, g}\right) /\left[w_{\text {rms }}\left(z_{i, g}\right)\right]^{3}$; and (d) buoyancy, $b_{\text {rms }}\left(z_{i, g}\right) / b_{\delta}$ (blue) and $b_{\delta} /\left[N^{2} \delta+N^{2}\left(z_{i, g}-z_{\text {enc }}\right)\right]$ (red). (e) Buoyancy scale inside the lower EZ sublayer, $\left[\langle b\rangle\left(z_{i, f}\right)-b_{\text {enc }}\right] /[\langle b\rangle$ $\left.\left(z_{i, g}\right)-b_{\text {enc }}\right]$. (f) Evolution of $\left(z_{10 \% \mathrm{mbf}}-z_{i, g}\right) / \delta$ (blue $)$ and $\left(z_{5 \% \mathrm{mbf}}-\right.$ $\left.z_{i, g}\right) / \delta$ (magenta), comparing the upper EZ limit definitions $z_{10 \% \mathrm{mb}}$, the height where the buoyancy flux is $10 \%$ of the minimum, and $z_{5} \%$ mbf, corresponding to $5 \%$, to the definition $z_{i, 1}=z_{i, g}+\delta$. Quasisteady behavior beginning at $z_{\text {end }} / L_{0} \simeq 10$ is observed for all quantities and the corresponding mean values are summarized in Table 3 . Light colors correspond to Re040, dark colors correspond to Re100.

$$
b_{\delta}=N^{2} \delta+\left[b_{0}\left(z_{i, g}\right)-\langle b\rangle\left(z_{i, g}\right)\right] \text {. }
$$

This expression allows us to interpret $b_{\delta}$, and therefore the maximum buoyancy rms (Fig. 4b), as a combination of two buoyancy increments.

The first contribution in Eq. (30), $N^{2} \delta$, can be interpreted, according to parcel theory, as the buoyancy force experienced by a parcel of fluid after a displacement $\delta$ from its neutral level across a region with buoyancy stratification $N^{2}$. Our numerical results show that the corresponding Richardson number

$$
\mathrm{Ri}_{\delta}=\frac{\delta\left(N^{2} \delta\right)}{w_{*}^{2}}=c_{\delta}^{2}
$$


TABLE 3. Constants defining the vertical structure of the entrainment zone, calculated within the entrainment equilibrium regime $\left(z_{\text {enc }} / L_{0} \geq 10\right)$.

\begin{tabular}{llllllll}
\hline & & $c_{\delta}$ & $c_{\varepsilon}$ & $c_{w 2}$ & $c_{b 1}$ & $c_{b 2}$ & $c_{b 3}$ \\
\hline Re040 & Mean & 0.53 & 0.68 & 0.19 & 0.39 & 0.47 & 0.43 \\
& $\sigma(\%)$ & 5.0 & 7.5 & 5.1 & 4.2 & 4.8 & 16 \\
$\operatorname{Re} 100$ & Mean & 0.52 & 0.51 & 0.23 & 0.39 & 0.55 & 0.44 \\
& $\sigma(\%)$ & 6.5 & 6.3 & 3.8 & 5.3 & 5.2 & 11 \\
\hline
\end{tabular}

is constant $\left(c_{\delta}^{2} \simeq 0.3\right)$. This constant behavior represents a continuous balance of potential and kinetic energy in the entrainment zone: on the one hand, if the buoyancy difference between the thermals and the environment is much stronger than $N^{2} \delta$ such that the thermals cannot penetrate into the stably stratified region anymore, then continuous buoyancy input from below will decrease this buoyancy difference until the thermals can overshoot again; on the other hand, if the buoyancy difference is much weaker than $N^{2} \delta$, the unhindered thermals will overshoot to a level of higher buoyancy, consequently steepening the mean buoyancy gradient across the upper portion of the EZ.

The second contribution in Eq. (30), the buoyancy difference $b_{0}\left(z_{i, g}\right)-\langle b\rangle\left(z_{i, g}\right)$, can be interpreted as the effective buoyancy increment with respect to $\langle b\rangle\left(z_{i, g}\right)$ that is felt by the nonthermal regions, whose buoyancy is better characterized by the original buoyancy profile $b_{0}$. The existence of two contributions to $b_{\delta}$ is consistent with the visualization (Figs. 6a,b and 7), because only a fraction of the upper EZ sublayer is occupied by penetrating thermals.

To predict $b_{\delta}$, Eq. (30), and thereby the mean buoyancy gradient and the rms of the buoyancy fluctuations inside the EZ, we still need an explicit parameterization of $\langle b\rangle$ $\left(z_{i, g}\right)$. From the previous observation that the mean state of the upper EZ sublayer seems to represent an average of thermal and nonthermal regions, we propose the relation

$$
b_{\delta}=c_{b 1}\left[N^{2} \delta+N^{2}\left(z_{i, g}-z_{\text {enc }}\right)\right] .
$$

This parameterization is validated in Fig. 8d. The constant is $c_{b 1} \simeq 0.4$ (see Table 3 ).

With this last step, we have obtained a complete parameterization of the buoyancy-related quantities within the upper EZ sublayer, given the controlling parameters $B_{0}$ and $N^{2}$. The buoyancy scale is parameterized as

$$
b_{\delta}=c_{b 1} b_{\mathrm{enc}}\left[C_{\mathrm{enc}, g}-1+\left(\delta / z_{\mathrm{enc}}\right)\right] \text {, }
$$

where $\delta / z_{\text {enc }}$ is given by Eq. (26) and $b_{\text {enc }}=N^{2} z_{\text {enc }}$ (see appendix B). The mean buoyancy at $z_{i, g}$, using Eq. (30), is parameterized as

$$
\langle b\rangle\left(z_{i, g}\right)=b_{\text {enc }}+\left[\left(1-c_{b 1}\right) / c_{b 1}\right] b_{\delta},
$$

the mean buoyancy gradient at $z_{i, g}$, from Eq. (22), is parameterized as

$$
b_{\delta} / \delta=c_{b 1} N^{2}\left[1+\left(C_{\text {enc }, g}-1\right)\left(\delta / z_{\text {enc }}\right)^{-1}\right],
$$

and the maximum rms of the buoyancy fluctuations is parameterized as

$$
b_{\mathrm{rms}}\left(z_{i, g}\right)=c_{b 2} b_{\delta},
$$

where $c_{b 2} \simeq 0.55$ (Fig. $8 \mathrm{~d}$ and Table 3).

We note that despite the relatively low Reynolds numbers $\operatorname{Re}_{\delta}=O\left(10^{2}\right)$ inside the EZ that we achieve in the simulations [Eq. (29)], the coefficients that are relevant for the discussion that follows, namely, $\left\{c_{\delta}, c_{b 1}\right.$, $\left.c_{b 2}\right\}$ and $c_{b 3}$ in section 6 , already show a relatively low Reynolds number dependence (Table 3). The largest variation between the two cases $\operatorname{Re} 040$ and $\operatorname{Re} 100$ occurs in $c_{b 2}$ and it is less than $15 \%$; for the other coefficients it is about $2 \%$. More importantly, we can differentiate between the variation of the mean and variance profiles of the buoyancy field that is caused by the temporal evolution of the EZ, and the variation due to Reynolds number effects. In particular, the rms of buoyancy fluctuation varies by a factor of 3 between $z_{\text {enc }} / L_{0}=10$ and $z_{\text {end }} / L_{0}=26$, whereas the change due to an increase by almost a factor of 3 in the Reynolds number between case Re040 and case Re100 is less than $15 \%$ (coefficient $c_{b 2}$ ).

\section{c. Discussion on the multiplicity of scales}

Although the length scale $\delta$ characterizes the thickness of the upper EZ sublayer and the integral length scale inside the turbulence pockets that exist in this sublayer, $\delta$ is not the only characteristic length scale within the entrainment zone. For example, the wavelength of the undulations along the horizontal directions are characterized by the CBL thickness, as observed from spectral and correlation analysis (not shown) and documented previously (see, e.g., de Roode et al. 2004). However, in this work we focus solely on $\delta$ because of its relevance for the buoyancy profiles, for the two-layer structure of the entrainment zone, and for the entrainment rate parameters (section 6).

We also point out that Eq. (32) can be written as

$$
b_{\delta}=c_{b 1} \delta b_{i},
$$

where 
$\delta b_{i}=b_{0}\left(z_{i, 1}\right)-b_{\text {enc }}=N^{2} \delta z_{i}$,

$\delta z_{i}=z_{i, g}+\delta-z_{\text {enc }}=z_{\text {enc }}\left[C_{\text {enc }, g}-1+\left(\delta / z_{\text {enc }}\right)\right]$,

and that we can interpret $\delta b_{i}$ and $\delta z_{i}$ as the buoyancy difference and EZ thickness definitions proposed and used in Fedorovich et al. (2004a) to parameterize the vertical profiles inside the entrainment zone according to the general structure model. However, there are notable differences. First, because of the clear definition of $\delta$, the definition of the EZ upper extent as $z_{i, 1}=z_{i, g}+\delta$ is equivalent to but more robust than the definition used by Fedorovich et al. (2004a) based on the zero-crossing (not shown because of large scatter in data), or based on a given fraction of the turbulent buoyancy flux (see Fig. $8 \mathrm{f}$ for a comparison). Second, we have shown that each of these two parameters $\delta b_{i}$ and $\delta z_{i}$ is composed of two characteristic scales that evolve differently in time as the CBL grows. Third, the self-similar behavior of the vertical profiles normalized using $\delta$ and $b_{\delta}$ is restricted to the upper EZ sublayer, and not the entire region of negative mean buoyancy flux.

This multilayer structure and the multiplicity of scales inside the EZ help to explain why difficulties were encountered in finding the appropriate scaling of the thickness for general structure models (Fedorovich and Mironov 1995; Fedorovich et al. 2004a), since these models rely on a single scale. This explanation seems to be more satisfactory than one based on gravity wave radiation, whose effect in the analysis of the EZ vertical structure has often been reported to be small (Deardorff et al. 1980; Zilitinkevich 1991; Fedorovich et al. 2004a).

It is worth emphasizing that $b_{\delta}$, or $\delta b_{i}$ as defined above, quantifies the variation of the mean buoyancy and buoyancy rms that results from an average between the crest regions with relatively large mean buoyancy gradient and the regions in between with relatively small buoyancy gradient (Figs. 6a,b). The buoyancy scales $b_{\delta}$ and $\delta b_{i}$ should not be interpreted as the buoyancy increment that the thermals feel or work against, which is better represented by $N^{2} \delta$ only. In other words, the CBL grows continuously against a constant stratification $N^{2}$, and not against an increasing buoyancy increment $\delta b_{i}$. (The decrease in time of the growth rate is due to the increasing CBL thickness over which the constant surface energy flux needs to be distributed, as quantified by the encroachment height, and not due to the increasing stratification $\delta b_{i}$.)

Last, the ratio between the EZ thickness, defined as $\delta z_{i}$, and the CBL thickness, $z_{\text {enc }}$, evolves according to

$$
\frac{\delta z_{i}}{z_{\text {enc }}}=C_{\text {enc }, g}-1+\left(\delta / z_{\text {enc }}\right) .
$$

The corresponding decrease in time toward the asymptotic value $C_{\text {enc, } g}-1 \simeq 0.24$ as $\delta / z_{\text {enc }}$ decreases was already found by Deardorff et al. (1980). However, as explained by those authors, the corresponding scaling was not well understood because neither the penetration depth $\delta$ nor the CBL thickness $z_{\text {enc }}$, separately, could explain the evolution of the ratio $\delta z_{i} / z_{\text {enc }}$. Here we show that the combination of both length scales, $\delta$ and $z_{\text {enc }}$, a consequence of the two-layer structure of the EZ, explains the observed behavior.

\section{The entrainment rate parameters}

The relation between the mean entrainment rate $w_{e}$, Eq. (13), and other entrainment rate parameters, like the minimum turbulent buoyancy flux and the buoyancy increment across the entrainment zone, has often been analyzed in the past within the framework of bulk models, like zero- or first-order models (see, e.g., Betts 1974; Sullivan et al. 1998; Fedorovich et al. 2004a, and references therein), and general structure models (Fedorovich and Mironov 1995; Fedorovich et al. 2004a). In contrast, the analysis presented in this section is based directly on the evolution equation for the mean buoyancy, without resorting to any particular model. The purpose is to understand better the behavior in time of those entrainment rate parameters, in particular, to understand how the twolayer structure discussed in the previous section affects this behavior, and thereby to provide reference data for model development.

Integrating in space the transport equation of the mean buoyancy deviation $\langle b\rangle-b_{0}$,

$$
\frac{\partial\left(\langle b\rangle-b_{0}\right)}{\partial t}=-\frac{\partial}{\partial z}\left(\left\langle b^{\prime} w^{\prime}\right\rangle-\kappa \frac{\partial\langle b\rangle}{\partial z}\right),
$$

from a given height $z_{i}(t)$ upward and applying the Leibniz rule yields

$$
\begin{aligned}
& \int_{z_{i}}^{z_{\infty}} \frac{\partial\left(\langle b\rangle-b_{0}\right)}{\partial t} d z \\
& \quad=\frac{d}{d t} \int_{z_{i}}^{z_{\infty}}\left(\langle b\rangle-b_{0}\right) d z+\left[\langle b\rangle\left(z_{i}, t\right)-b_{0}\left(z_{i}\right)\right] \frac{d z_{i}}{d t} \\
& \quad=\int_{z_{i}}^{z_{\infty}} \frac{\partial}{\partial z}\left(\kappa \frac{\partial\langle b\rangle}{\partial z}-\left\langle b^{\prime} w^{\prime}\right\rangle\right) d z
\end{aligned}
$$

The analysis of the mean buoyancy as a deviation from the reference background profile $b_{0}$, instead of just $\langle b\rangle$, has the advantage that the result is independent of the upper limit of integration $z_{\infty}$ when it is located far enough into the nonturbulent stably stratified region. Dividing by $B_{0}$, we obtain the entrainment rate equation 


$$
\frac{1}{B_{0}} \Delta b_{i} \frac{d z_{i}}{d t}=A
$$

where

$$
\Delta b_{i}(t)=b_{0}\left(z_{i}\right)-\langle b\rangle\left(z_{i}, t\right)
$$

and

$$
A(t)=A_{t}(t)+A_{m}(t)+A_{d}(t) .
$$

The term $\Delta b_{i}$ is the buoyancy deviation of the current mean buoyancy from the background reference $b_{0}(z)$ at the height $z_{i}(t)$. The normalized entrainment flux, $A$, also known as entrainment ratio, is composed of three terms,

$$
\begin{aligned}
A_{t}(t) & =-\frac{\left\langle b^{\prime} w^{\prime}\right\rangle\left(z_{i}\right)}{B_{0}}, \\
A_{d}(t) & =-\frac{1}{B_{0}} \frac{d}{d t} \int_{z_{i}}^{z_{\infty}}\left(b_{0}-\langle b\rangle\right) d z, \\
A_{m}(t) & =\frac{\kappa}{B_{0}} \frac{\partial\langle b\rangle}{\partial z}\left(z_{i}\right)-\mathrm{Re}_{0}^{-1},
\end{aligned}
$$

corresponding, respectively, to the turbulent contribution, the distortion or shape contribution due to the EZ's finite thickness, and the molecular contribution (which includes the molecular contribution of the background stratification as $\mathrm{Re}_{0}^{-1}$ ).

The previous equation and definitions can be applied at any CBL-top height $z_{i}$. For the rest of this section, we particularize them at $z_{i}=C_{\text {enc }, f} z_{\text {enc }}$, the height of minimum buoyancy flux, to be consistent with the usual definition of the entrainment ratio $A$. We use the smooth approximation $C_{\text {enc } f} z_{\text {enc }}$ to the instantaneous value $z_{i, f}$, valid for $z_{\text {end }} / L_{0} \geqslant 10$ (see section $4 \mathrm{a}$ ), in order to reduce the variability in the calculation of the time derivative in the distortion or shape contribution $A_{d}$; the corresponding effect on the calculated values of $A$ and $\Delta b_{i, f}$ is less than $5 \%$.

\section{a. Contributions to the entrainment ratio}

The largest contribution to the entrainment ratio $A$ is the turbulent term $A_{t}$ (Fig. 9). On the one hand, the growth of $A_{t}$ by about a factor of 2 as the CBL develops in time between $z_{\text {end }} / L_{0}<10$ and $z_{\text {end }} / L_{0}>20$ is consistent with the factor of 2 variation observed by Fedorovich et al. (2004a) in their LES data when the strength of the stratification is increased from the weak stratification, corresponding to the CBL state $z_{\text {end }} / L_{0} \simeq$ 7, to the strong stratification, corresponding to the $\mathrm{CBL}$ state $z_{\text {end }} / L_{0} \simeq 23$ (see section 3 ). However, the DNS

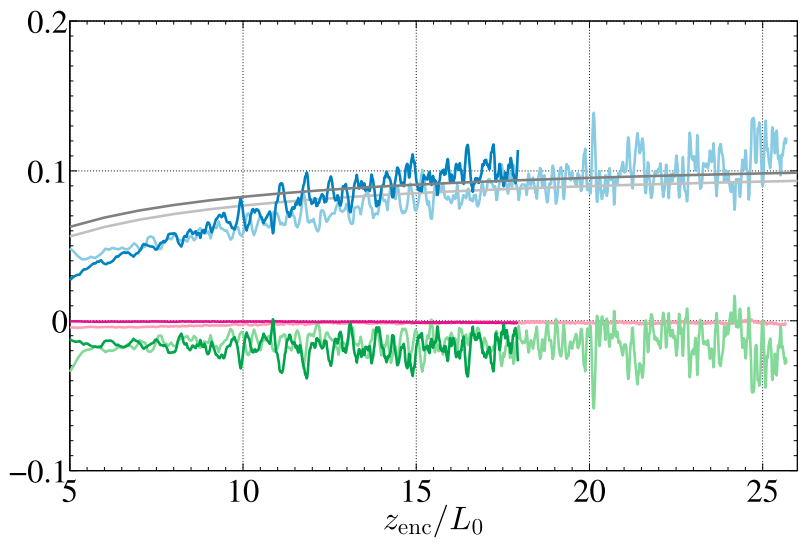

FIG. 9. Temporal evolution of the contributions to the entrainment ratio $A$ measured at $z_{i, f}$, Eq. (45): $A_{t}$ (blue), $A_{m}$ (magenta), and $A_{d}$ (green). The gray line is the parameterization of $A_{t}$ in Eq. (50). Light colors correspond to Re040, dark colors correspond to Re100.

values $A_{t} \lesssim 0.1$ at $z_{\text {end }} / L_{0} \simeq 20$ are systematically smaller than the interval $0.1-0.15$ reported by those authors. Since the Reynolds number effect on $A_{t}$ is less than $15 \%$ (Fig. 9) and both cases consider a CBL growing into a linearly stratified atmosphere, this difference is likely due to subgrid-scale model effects inside the entrainment zone. On the other hand, values of $A_{t}$ smaller than DNS values were observed in the convective tank experiments by Deardorff et al. (1980), despite the larger $z_{\text {end }} / L_{0}$, about 50. This apparent discrepancy is possibly due to the relatively small reference Reynolds number in those tank experiments, $\mathrm{Re}_{0} \simeq 10$ instead of $\mathrm{Re}_{0} \simeq 100$ here, which in turn implies a relatively small Reynolds number inside the entrainment zone in the tank experiments and a tendency to have a thicker EZ and a less pronounced minimum in the profile of the turbulent buoyancy flux.

The second contribution to the entrainment ratio is the distortion or shape term $A_{d}$. Its value is relatively small compared to the contribution of the turbulent buoyancy flux $A_{t}$. On average, we observe

$$
A_{d} \simeq-0.02
$$

whereas $A_{t} \simeq 0.1$. This result is in contrast with the conclusion of Sullivan et al. (1998), which states that both terms are comparable to each other for CBLs with relatively thick EZ. We take note, however, that the buoyancy term $\Delta b_{i, f}$ in Eq. (42), and thereby $A_{d}$, are defined here differently, namely as a local deviation with respect to the background profile $b_{0}(z)$, and not as a global buoyancy increment across the whole entrainment zone (Fig. 10).

In contrast to $A_{t}$, the distortion or shape term $A_{d}$ is approximately constant in time (Fig. 9). This steady behavior can be understood from the two-layer vertical 


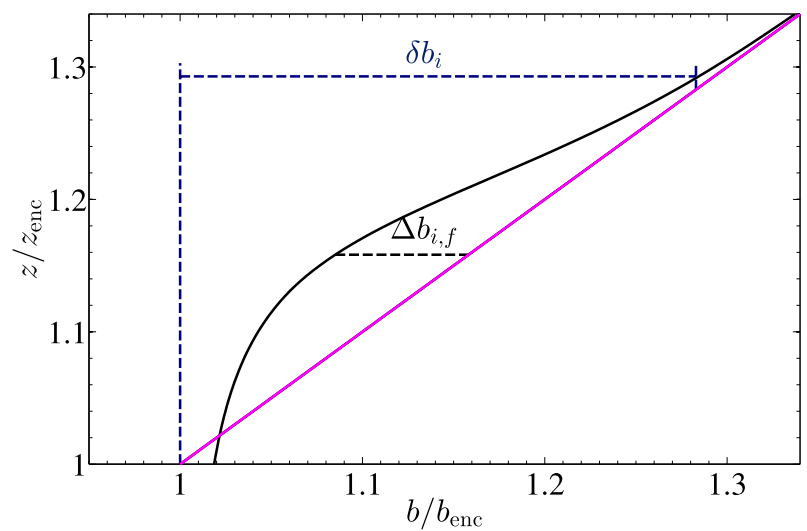

FIG. 10. Sketch illustrating the difference between the local buoyancy increment $\Delta b_{i, f}$, Eq. (43), evaluated at $z_{i}=z_{i, f}$, and the global buoyancy difference $\delta b_{i}$ across the whole entrainment zone, Eq. (38).

structure of the EZ that is described in the previous section. The integral in Eq. (45) can be split into two integrals: one integral from $z_{i, f}$ to $z_{i, g}$ and another integral from $z_{i, g}$ to $z_{\infty}$. It is then easy to show from the scalings derived in the previous section that this second contribution from the upper EZ sublayer continuously decreases, whereas the first contribution from the lower EZ sublayer is indeed constant in time. This latter contribution, according to Fig. 9, seems to dominate the evolution of $A_{d}$.

Last, the molecular contribution to the entrainment ratio, $A_{m}$, is already negligibly small for the Reynolds numbers achieved in this work (Fig. 9).

\section{b. The local buoyancy increment}

We have learned before that the contribution to the entrainment ratio $A$ from the finite EZ thickness through the distortion term $A_{d}$ is relatively small, about $20 \%$ or less. However, the finite EZ thickness still remains important because the smooth variation of the mean buoyancy inside the entrainment zone over a finite EZ thickness determines a local buoyancy increment $\Delta b_{i, f}$ in Eq. (42) that is significantly smaller than the global buoyancy increment $\delta b_{i}$ across the whole entrainment zone (see Fig. 10). We can quantify this difference as follows.

As a first approximation, we can assume that the mean buoyancy inside the lower EZ sublayer, in particular $\langle b\rangle(z)-b_{\text {enc }}$, tends toward a steady profile when normalized with $\langle b\rangle\left(z_{i, g}\right)-b_{\text {enc. }}$. The reason is that the lower EZ sublayer is relatively well mixed and approximately quasi-steady in the entrainment equilibrium regime (see section $4 \mathrm{~b}$ ): the top and bottom values $\langle b\rangle\left(z_{i, g}\right)$ and $\langle b\rangle\left(z_{i, 0}\right) \simeq b_{\text {enc }}$ (see appendix B) vary in time, but turbulence mixes the buoyancy across that region relatively fast. Hence, we can hypothesize that

$$
\langle b\rangle\left(z_{i, f}\right)-b_{\mathrm{enc}}=c_{b 3}\left[\langle b\rangle\left(z_{i, g}\right)-b_{\mathrm{enc}}\right],
$$

since the height of minimum buoyancy flux $z_{i, f}$ is approximately in the middle of the lower EZ sublayer (see Figs. $4 \mathrm{~b}$ and 7). Figure 8e supports this relation for $z_{\text {end }}$ $L_{0}>10-15$, the constant of proportionality being $c_{b 3} \simeq$ 0.45 (see Table 3).

Combining this result with Eqs. (33) and (34) yields

$$
\Delta b_{i, f} / b_{\mathrm{enc}}=\beta_{0}-\beta_{1}\left(\delta / z_{\mathrm{enc}}\right),
$$

where

$$
\begin{aligned}
& \beta_{0}=C_{\text {enc }, f}-1-c_{b 3}\left(1-c_{b_{1}}\right)\left(C_{\text {enc }, g}-1\right), \\
& \beta_{1}=c_{b 3}\left(1-c_{b_{1}}\right)
\end{aligned}
$$

and $\delta / z_{\text {enc }}$ is given by Eq. (26). Based on the constants in Tables 2 and $3, \beta_{0} \simeq 0.086$ and $\beta_{1} \simeq 0.27$. Comparing with $\delta b_{i}$, Eq. (38), we observe that $\Delta b_{i, f}$ tends asymptotically toward a constant fraction $\simeq 0.26$ of $\delta b_{i}$. However, the ratio $\Delta b_{i, f} / \delta b_{i}$ increases in time by a factor of 2 during the intermediate states $z_{\text {end }} / L_{0} \simeq 10-26$, when the contribution of $\delta$ to the EZ structure is not negligible.

\section{c. Asymptotic behavior of $A$}

Substituting Eq. (48) in Eq. (42) and using Eq. (14), we obtain an explicit expression for the entrainment ratio in the form

$$
A_{t}=\gamma_{0}-\gamma_{1}\left(\delta / z_{\text {enc }}\right),
$$

where

$$
\begin{aligned}
& \gamma_{0}=C_{\text {enc } f f} \beta_{0}-A_{d}, \\
& \gamma_{1}=C_{\text {enc } f} \beta_{1} .
\end{aligned}
$$

From the previous section, we obtain the estimates $\gamma_{0} \simeq$ 0.12 and $\gamma_{1} \simeq 0.31$. The good agreement of this parameterization with the DNS data (Fig. 9) is mainly a consequence of the good prediction of the local buoyancy increment by Eq. (48), since Eq. (50) follows from the exact relation, Eq. (42). Hence, the evolution of $A$ in time is a consequence of the evolution of the two-layer structure of the EZ. We can also infer that, for a CBL penetrating into a linearly stratified atmosphere, $A_{t}$ tends toward an asymptotic value $\simeq 0.12$, within an accuracy of $\simeq 15 \%$ (Table 3 ). This value is significantly below the entrainment ratio value $\simeq 0.2$ that works well for predicting the mean entrainment rate using the zero-order bulk model. However, as stated in the 
introduction and emphasized by Fedorovich et al. (2004a), there is no inconsistency between both results because the zero-order model is not designed to capture the effects of the EZ's finite thickness.

The evolution of $A_{t}$ according to Eq. (50) can be interpreted in terms of the dominant balance in the turbulence kinetic energy equation Eq. (16), particularized at $z_{i, f}$, between the turbulent transport, and the turbulent buoyancy flux and the viscous dissipation (Fig. 3b). The three terms are expected to follow an inviscid scaling in terms of the local integral length and velocity scales of the turbulence. Since $z_{i, f}$ is still relatively close to the upper EZ sublayer for the interval $z_{\text {end }} / L_{0} \simeq 10-26$ (see Fig. 4b), we can also anticipate that the integral length and velocity scales that are observed at $z_{i, f}$ are a combination of the corresponding scales within each of the two EZ sublayers within that interval of time. Although the velocity scales in both EZ sublayers are proportional to the convective velocity, the length scales are different: a constant fraction of $z_{\text {enc }}$ in the lower EZ sublayer and $\delta$ in the upper EZ sublayer. If we propose

$$
-\left\langle b^{\prime} w^{\prime}\right\rangle\left(z_{i, f}\right)=\gamma_{0} \frac{w_{*}^{3}}{z_{\mathrm{enc}}+\left(\gamma_{1} / \gamma_{0}\right) \delta}
$$

and expand the fraction in terms of the small number $\left(\gamma_{1} / \gamma_{0}\right)\left(\delta / z_{\text {enc }}\right)$, we recover Eq. (50) as a first approximation. Hence, the combined effect of the two-layer structure on the local energetics inside the entrainment zone, Eq. (16), including the turbulent buoyancy flux, can be interpreted in terms of an average length scale proportional to $z_{\text {enc }}+\left(\gamma_{1} / \gamma_{0}\right) \delta$, where $\gamma_{1} / \gamma_{0} \simeq 1.3$. As the CBL develops in time and the ratio $\delta / z_{\text {enc }}$ decreases, the upper EZ sublayer recedes toward $z_{i, g}$ and the length scale that remains effective at $z_{i, f}$ is that of the lower EZ sublayer, namely $z_{\text {enc }}$.

\section{d. The entrainment rate-Richardson number power law}

The entrainment rate equation, Eq. (42), is sometimes expressed as a relation between a nondimensional or normalized mean entrainment rate $E=w_{e} / w_{*}$ and a Richardson number Ri. Different power laws $E \propto \mathrm{Ri}^{-n}$ have been proposed in the literature, although scatter in the data and uncertainty in the exponent $n$ still prevent us from reaching a definite conclusion, in particular for intermediate values of Ri (see, e.g., Zilitinkevich 1991; Fedorovich et al. 2004a; Jonker et al. 2012, and references therein). For the case of a CBL growing into a linearly stratified fluid, the different choices for the CBL-top height $z_{i, \xi}$ that is used in the definition of $w_{e}$ and $w_{*}$ can only explain a relatively small variation in the proportionality coefficient of this relation, since all of these heights become commensurate with each other beyond $z_{\text {end }} / L_{0} \simeq 5-10$ (section $\left.4 \mathrm{a}\right)$. In contrast, the particular buoyancy scale that is used to define the Richardson number can affect the functional relation between $E$ and Ri more significantly. This section is devoted to this issue.

Conventionally, a convective Richardson number is defined as

$$
\mathrm{Ri}_{*}=\frac{z_{\text {enc }} \delta b_{i}}{w_{*}^{2}}
$$

where $\delta b_{i}$ [Eq. (38)] is a measure of the buoyancy variation across the whole entrainment zone (Deardorff et al. 1980; Sullivan et al. 1998; Fedorovich et al. 2004a). Combining this definition with Eq. (14), we obtain the relation

$$
E=\alpha \mathrm{Ri}_{*}^{-1},
$$

where

$$
\alpha=C_{\text {enc } f}\left[C_{\text {enc }, g}-1+\left(\delta / z_{\text {enc }}\right)\right] .
$$

[The prefactor $C_{\mathrm{enc}, f}$ in the expression above results from calculating the entrainment velocity at $z_{i, f}$ and computing $w_{*}$ according to Eq. (9)—as already mentioned, other choices vary this prefactor merely by a constant of order one and its particular value is irrelevant for the discussion that follows.] This analytic result has two important implications.

First, asymptotically, the proportionality coefficient $\alpha(t)$ approaches $\alpha_{0}=C_{\text {enc, } f}\left(C_{\text {enc, } g}-1\right)$ and hence $E \propto \mathrm{Ri}_{*}^{-1}$. This is one of the power laws proposed in the literature based on the estimate $\left\langle b^{\prime} w^{\prime}\right\rangle\left(z_{i}\right) \propto w_{*}^{3} / z_{i} \simeq B_{0}$ for the turbulent flux within the entrainment zone and the approximation $w_{e} \delta b \simeq-\left\langle b^{\prime} w^{\prime}\right\rangle\left(z_{i}\right)$ [for more details, see, e.g., Fernando (1991)]. However, for the interval $\mathrm{Ri}_{*} \simeq 8-23\left(z_{\text {end }} / L_{0} \simeq 10-26\right)$ considered in this study, which is representative of atmospheric conditions (see section 3), the evolution of the normalized mean entrainment rate $E$ deviates from that asymptotic limit: a steeper curve is observed in Fig. 11, in agreement with previous results that suggested exponents $n$ different from 1 (Turner 1973; Deardorff et al. 1980; Fedorovich et al. 2004a). Our results indicate that these deviations stem from the effect that the upper EZ sublayer has on the entrainment rate parameters, specifically, the term $\delta / z_{\text {enc }}$ in Eq. (55). When we express $\delta / z_{\text {enc }}$ as a function of $\mathrm{Ri}_{*}$ using Eq. (38),

$$
\delta / z_{\mathrm{enc}}=\frac{c_{\delta}^{2}}{2 \mathrm{Ri}_{*}}\left\{1+\left[4 c_{\delta}^{-2}\left(C_{\mathrm{enc}, g}-1\right) \mathrm{Ri}_{*}+1\right]^{1 / 2}\right\}
$$

we obtain the approximation 


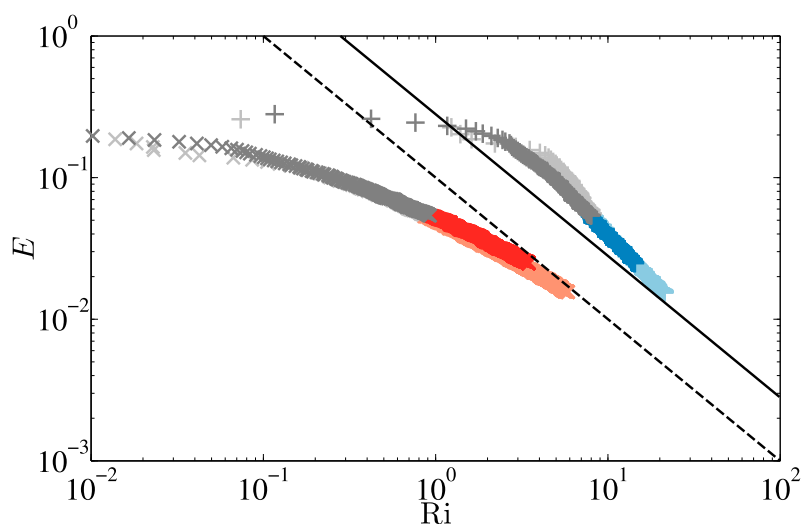

FIG. 11. Scatterplot of the normalized entrainment rate $E=$ $w_{e} / w_{*}$ against the Richardson numbers $\mathrm{Ri}_{*}=z_{\text {enc }} \delta b_{i} / w_{*}^{2}$ and $\mathrm{Ri}_{i, f}=z_{\text {enc }} \Delta b_{i, f} / w_{*}^{2}$. Gray denotes data from earlier time $z_{\text {end }} / L_{0}<$ 10. For $z_{\text {end }} / L_{0} \geq 10$, blue corresponds to $\mathrm{Ri}_{*}$ and red to $\mathrm{Ri}_{i, f}$. The solid black line is $0.28 \mathrm{Ri}_{*}^{-1}$, based on the asymptotic behavior of $\alpha(t)$ toward 0.28 . The dashed black line is $0.10 \mathrm{Ri}_{i, f}^{-1}$, based on the asymptotic behavior of $A(t)$ toward 0.10 . Light colors correspond to Re 040 , dark colors correspond to Re100.

$$
E \simeq\left(\alpha_{0}+\alpha_{1} \mathrm{Ri}_{*}^{-1 / 2}+\alpha_{2} \mathrm{Ri}_{*}^{-1}\right) \mathrm{Ri}_{*}^{-1},
$$

valid for $\mathrm{Ri}_{*} \gg c_{\delta}^{2} /\left[4\left(C_{\mathrm{enc}, g}-1\right)\right] \simeq 0.28$, where

$$
\begin{aligned}
& \alpha_{0}=C_{\text {enc }, f}\left(C_{\text {enc }, g}-1\right), \\
& \alpha_{1}=C_{\text {enc }, f}\left(C_{\text {enc }, g}-1\right)^{1 / 2} c_{\delta}, \\
& \alpha_{2}=C_{\text {enc }, f} c_{\delta}^{2} / 2 .
\end{aligned}
$$

From the constants in Tables 2 and 3, we obtain $\alpha_{0} \simeq$ $0.28, \alpha_{1} \simeq 0.29$, and $\alpha_{2} \simeq 0.16$. Equation (57) helps to explain the different scalings $E \propto \mathrm{Ri}_{*}^{-n}, 1 \leq n<2$, found in the literature for intermediate values of the convective Richardson number $\mathrm{Ri}_{*}$. Equation (57) also allows us to estimate the error in predicting $E$ according to $E \simeq \alpha_{0} \mathrm{Ri}_{*}^{-1}$ : even at the strong stratification limit $z_{\text {end }}$ l $L_{0} \simeq 50\left(\mathrm{Ri}_{*} \simeq 50\right)$, this error is still larger than $10 \%$. The question still remains, though, whether we could interpret Eq. (57) and the effect of the upper EZ sublayer on $\alpha$ in terms of some of the mixing mechanisms that have been proposed to play a role at the entrainment zone, in particular those associated with the interaction of turbulence and gravity waves (Carruthers and Hunt 1986; Fernando 1991).

It is worth noting that an alternative definition of a Richardson number as $\mathrm{Ri}_{N, \xi}=N^{2} z_{i, \xi}^{2} / w_{*, \xi}^{2}$, where $w_{*, \xi}=$ $\left(B_{0} z_{i, \xi}\right)^{1 / 3}$, leads to the exact relation $w_{e, \xi} / w_{*, \xi}=$ $C_{\text {enc }, \xi}^{2} \mathrm{Ri}_{N, \xi}^{-1}$ once $z_{i, \xi}$ becomes proportional to $z_{\text {enc }}$, which occurs at about $z_{\text {end }} / L_{0} \simeq 10$ (see section $4 \mathrm{a}$ ). The range $z_{\text {end }} / L_{0} \simeq 10-26$ considered in our study corresponds to $N^{2} z_{\text {enc }}^{2} / w_{*}^{2} \simeq 21-77$. Hence, a very clear relation
$E \propto \mathrm{Ri}^{-1}$ appears much earlier than when using the convection Richardson number $\mathrm{Ri}_{*}$. However, $\mathrm{Ri}_{N, \xi}$ does not reflect the evolution of the local dynamics inside the EZ during the intermediate range of atmospheric $\mathrm{Ri}$ chardson numbers considered in this study.

Last, by comparing cases Re040 and Re100 we also observe in Fig. 11 that Reynolds number effects in the functional relation $E=f\left(\mathrm{Ri}_{*}\right)$ are negligibly small beyond $\mathrm{Re}_{*} \simeq 10^{3}$, which is the value attained in simulation $\operatorname{Re} 040$ at $z_{\text {end }} / L_{0} \simeq 10$. This result agrees with previous data (see, e.g., Fernando and Little 1990; Jonker and Jimenez 2014). [Prandtl numbers greater than 1 , not considered in this study, might affect this mixing transition into an inviscid behavior (see, e.g., Jonker et al. 2012)].

The second implication of Eq. (55) is that the proportionality coefficient $\alpha$ is different from the entrainment ratio $A$. It is not only different by a proportionality constant of order one, but it also evolves differently in time, since $A(t)$ increases (Fig. 9) and $\alpha(t)$ decreases (since $\delta / z_{\text {enc }}$ decreases). If desired, a functional relation in which the proportionality coefficient is the entrainment ratio $A(t)$ can be obtained by rewriting Eq. (42) as

$$
E=A \mathrm{Ri}_{i, f}^{-1} .
$$

Similar to the previous observation, a strong deviation during the interval $z_{\text {enc }} / L_{0} \simeq 10-26$ from the asymptotic behavior $E \propto \mathrm{Ri}_{i, f}^{-1}$ is exhibited in Fig. 11. This deviation is again due to the upper EZ sublayer, in this case, due to its effect on the evolution of $A$ toward its asymptotic value, Eq. (50). However, the Richardson number

$$
\mathrm{Ri}_{i, f}=\frac{z_{\text {enc }} \Delta b_{i, f}}{w_{*}^{2}}
$$

is now based on the local buoyancy increment $\Delta b_{i, f}$ characterizing the entrainment rate equation at $z_{i, f}$, and not on the buoyancy increment $\delta b_{i}$ as used in the definition of the convective Richardson number $\mathrm{Ri}_{*}$. Hence, Eq. (42), which is derived from first principles, reduces the degree of freedom to choose the proportionality coefficient and the Richardson number in the entrainment rate equation $E \propto \mathrm{Ri}^{-n}$ : if we choose the proportionality coefficient to be the usual entrainment ratio $A$ defined as Eq. (44), then the exact equation imposes a corresponding Richardson number defined with a local buoyancy increment $\Delta b_{i, f}$. On the other hand, if we simply relate the normalized mean entrainment rate $E$ to the convective Richardson number $\mathrm{Ri}_{*}$, then the exact equation imposes a corresponding proportionality coefficient $\alpha$ that is different from the entrainment ratio $A$. 


\section{Conclusions}

In this paper, we analyzed the vertical structure of the entrainment zone (EZ) and its effect on the entrainment rate parameters using data from direct numerical simulations of a convective boundary layer (CBL) growing into a linearly stratified environment.

Regarding the vertical structure of the entrainment zone, we found that it is better described by two overlapping sublayers: an upper EZ sublayer dominated by the penetrating thermals that are directly affected by the stratification $N^{2}$, and a lower EZ sublayer dominated by the troughs of mixed fluid that are only indirectly affected by the stratification $N^{2}$ through its effect on the evolution in time of the CBL thickness. Consequently, the two regions are characterized by different length scales. For the upper EZ sublayer, we argued that $\delta$, defined as the gradient thickness based on the maximum buoyancy gradient, is the characteristic vertical length, since scaling with it leads to a self-similar behavior of the mean and rms buoyancy profiles within that part of the entrainment zone, whereas scaling with the CBL thickness $z_{i}$ does not. Physically, we interpret $\delta$ as the mean penetration depth of an overshooting thermal, and such interpretation is supported by the agreement of $\delta$ 's evolution in time with the prediction from parcel theory, namely, $\delta \propto\left(w_{*} / N\right)$, where $w_{*}$ is the convective velocity. We also found that $\delta$ is at the same time the integral length scale of the turbulence inside those crest regions, since the viscous dissipation rate $\varepsilon$ at the height of maximum gradient $z_{i, g}$ scales as $\varepsilon\left(z_{i, g}\right) \propto\left[w_{\text {rms }}\left(z_{i, g}\right)\right]^{3} / \delta$. Within the lower EZ sublayer, the characteristic length scale is transitioning from $\delta$ to $z_{i}$ as one approaches the mixed layer. Correspondingly, different buoyancy scales are found, which reflects on the buoyancy fluctuations being a combination of the buoyancy increment associated with the penetrating thermal, and the buoyancy increment associated with the nonthermal regions that mainly retain the original stratification $N^{2}$. Parameterizations for the characteristic scales are provided, which allows for the reconstruction of the vertical profiles of the mean and variance of the buoyancy field within the EZ at any time within the equilibrium entrainment regime.

These findings justify the consideration for a second turbulence length scale for turbulence models at the EZ, one that is different from $z_{i}$ and behaves according to the parcel theory prediction. This multiplicity of scales inside the EZ also explains difficulties found in previous analyses that considered the entrainment zone as a single layer with vertical profiles characterized by a single set of characteristic scales.

To analyze the effect of the vertical structure of the $\mathrm{EZ}$ on the entrainment rate parameters, we derived an exact equation for the mean entrainment rate at the height of minimum buoyancy flux, $z_{i, f}$, whose terms depend neither on the definition of the upper extent of the entrainment zone nor on any bulk model formulation. We obtained this equation from an integral analysis of the evolution equation for the mean buoyancy. We found that the direct contribution to the entrainment ratio $A$ from the EZ finite thickness through the distortion term is small, but the EZ finite thickness also decreases the local buoyancy increment associated with the exact equation, which then compensates for the small $A$. For the case of a CBL growing into a linearly stratified atmosphere, we provided parameterizations for the local buoyancy increment, and both turbulent and distortion contributions to $A$. Based on the parameterization of the turbulent contribution $-\left\langle b^{\prime} w^{\prime}\right\rangle\left(z_{i, f}\right) /$ $B_{0}$, we found that this ratio asymptotes in time to $\simeq 0.12$.

Regarding the relation between the normalized mean entrainment rate $E$ and the convective Richardson number $\mathrm{Ri}_{*}$, we show that the deviation from the power-law with exponent -1 under typical atmospheric conditions is explained by the effect of the upper EZ sublayer on the buoyancy increment across the whole EZ and on the corresponding proportionality coefficient $\alpha$. As the upper EZ sublayer becomes thinner relative to the CBL, $\alpha$ asymptotes to a constant $\simeq 0.28$ and the functional relation between the normalized mean entrainment rate $E$ and the convective Richardson number $\mathrm{Ri}_{*}$ approaches a powerlaw behavior with exponent -1 . This finding shows that the deviation is apparently not due to the radiation of gravity waves, confirming previous indications. We noted also that the proportionality coefficient $\alpha$ evolves in time differently from the entrainment ratio $A$, implying that an inappropriate $\{A, \mathrm{Ri}\}$ pair could partially explain the failure of previous attempts to relate $E$ to a certain Richardson number, $\mathrm{Ri}$, through a power law.

Acknowledgments. The authors thank A. de Lózar, C. van Heerwaarden, and B. Stevens for insightful discussions on the topic. Prof. E. Fedorovich is gratefully acknowledged for comments and constructive criticism that helped to improve the manuscript stylistically and scientifically. Support from the Max Planck Society via its Max Planck Research Groups programme is gratefully acknowledged. Computational time was provided by the Jülich Supercomputing Centre.

\section{APPENDIX A}

\section{Minimum CBL Thickness}

The length scale $L_{0}=\left(B_{0} / N^{3}\right)^{1 / 2}$ can also be related to the minimum boundary layer thickness at which 


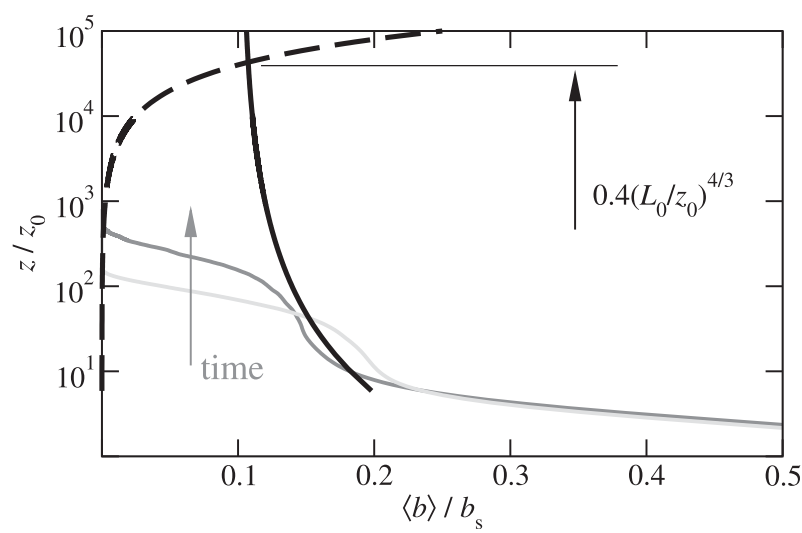

FIG. A1. Sketch illustrating the crossover height $\left[0.4\left(L_{0} / z_{0}\right)^{1 / 3}\right]$ $L_{0}=\left[0.4\left(L_{0} / z_{0}\right)^{4 / 3}\right] z_{0}$ at which the background buoyancy profile $b_{0}=$ $N^{2} z$ (dashed line) is felt by the growing boundary layer (solid lines). The solid black line is $\langle b\rangle / b_{s} \simeq 0.10+0.17\left(z / z_{0}\right)^{-1 / 3}$, as obtained from DNS of a neutral CBL (Mellado 2012, gray profiles); $b_{s}$ is the surface mean buoyancy. The background stratification shown in this figure corresponds to the case of a smooth wall, $\nu / \kappa=1$ and $\operatorname{Re}_{0}=10^{5}, z_{0}$ being then equal to the diffusive length $\left(\kappa^{3} / B_{0}\right)^{1 / 4}$.

stratification affects the evolution and the morphology of the boundary layer, and the system changes regimes. We can identify three different regimes. First, there is an early regime in which the turbulent boundary layer is shallow enough to behave essentially as if it were developing in neutral conditions: the boundary layer height varies proportionally to $\left(B_{0} t^{3}\right)^{1 / 2}$ and the kinetic energy profiles behave self-similarly when normalized by the convection scales (Mellado 2012). Second, when the boundary layer thickness becomes comparable to $L_{0}$, there appears an intermediate regime in which $N^{2}$ becomes relevant, turbulence kinetic energy is increasingly transferred from the vertical to the horizontal direction, and the growth rate diminishes with respect to that of the early regime. Eventually, the equilibrium (quasi-steady) entrainment regime is achieved, in which the CBL thickness varies proportionally to $\left(B_{0} t / N^{2}\right)^{1 / 2}$ and the kinetic energy profiles become again self-similar, at least approximately, when normalized with the convection scales (see section $4 \mathrm{~b}$ and also appendix B). The physical interpretation of $L_{0}$ within this context of regime transition is explained as follows.

For the neutral case $N^{2}=0$, the mean buoyancy profile tends toward $\alpha_{1} b_{s}$ as the distance to the surface increases (Fig. A1). This buoyancy level $\alpha_{1} b_{s}$ is a constant fraction of the surface mean buoyancy $b_{s}=\alpha_{2}\left(B_{0}^{2} / z_{0}\right)^{1 / 3}$, where $z_{0}$ indicates either the roughness length or the diffusive length, depending on the surface properties (Garratt 1992). The height at which this buoyancy level $\alpha_{1} b_{s}$ becomes comparable with that of the background reference profile $b_{0}=N^{2} z$ yields the crossover height $\alpha_{1} b_{s} / N^{2}=\alpha_{1} \alpha_{2} N^{-2}\left(B_{0}^{2} / z_{0}\right)^{1 / 3}$. When the depth of the turbulent boundary layer is much smaller than this crossover height, the turbulent boundary layer is not affected by the stratification aloft. Hence, this crossover height can be considered as the minimum CBL thickness at which the transition of regimes (described in the previous paragraph) occurs, and it can be expressed explicitly in terms of $L_{0}$ as $\left[\alpha_{1} \alpha_{2}\left(L_{0} / z_{0}\right)^{1 / 3}\right] L_{0}$.

For a smooth surface and in the case of $\operatorname{Pr}=\nu / \kappa=1$, as considered in this work, the diffusive length is $z_{0}=$ $\left(\kappa^{3} / B_{0}\right)^{1 / 4}$ and thus $L_{0} / z_{0}=\mathrm{Re}_{0}^{3 / 4}$, where $\operatorname{Re}_{0}=B_{0} /\left(\nu N^{2}\right)$. Moreover, $\alpha_{1} \simeq 0.1$ and $\alpha_{2} \simeq 4$ (Mellado 2012). Hence, in terms of the controlling parameters of the problem, the minimum CBL thickness is $\simeq 0.4 L_{0} \operatorname{Re}_{0}^{1 / 4}$, that is, basically proportional to $L_{0}$ because of the weak dependence on the Reynolds number as $\mathrm{Re}_{0}^{1 / 4}$.

\section{APPENDIX B}

\section{The Encroachment and Convective Scales}

For the well-mixed layer statistics, the encroachment buoyancy (Carson and Smith 1975)

$$
b_{\text {enc }}=N^{2} z_{\text {enc }}
$$

and the convective scales (Deardorff 1970)

$$
b_{*, \xi}=\left(z_{i, \xi} / B_{0}^{2}\right)^{-1 / 3}, w_{*, \xi}=\left(B_{0} z_{i, \xi}\right)^{1 / 3}
$$

are well-known characteristic scales of the mean buoyancy, and the variances of the turbulent fluctuations of buoyancy, $\left\langle b^{\prime} b^{\prime}\right\rangle$, and velocity components $\left\langle w^{\prime} w^{\prime}\right\rangle$ and $\left\langle v_{1}^{\prime} v_{1}^{\prime}\right\rangle$, respectively. For the convective scales, we will use $z_{i, \xi}=z_{\text {enc }}$, (denoting the corresponding convective scales as $b_{*}=B_{0} / w_{*}$ and $\left.w_{*}\right)$ since the variation in the magnitude of the convective scales when other definitions of the CBL-top height $z_{i, \xi}$ are used is only at most $7 \%$ (Table 2).

Figure B1 shows that the normalized vertical profiles of the mean buoyancy, the mean buoyancy gradient, the buoyancy flux, and the variances of buoyancy and the vertical and horizontal velocity components exhibit the expected shapes of these profiles (Stull 1973; Deardorff et al. 1980) and that within the mixed layer, the profiles at different times tend to collapse on top of each other within the achieved statistical convergence.

Figure B2 shows in more detail the tendency of the mixed layer statistics toward proportionality with the corresponding encroachment and convective scales, which also facilitates the quantification of possible low Reynolds number effects. The following three features 

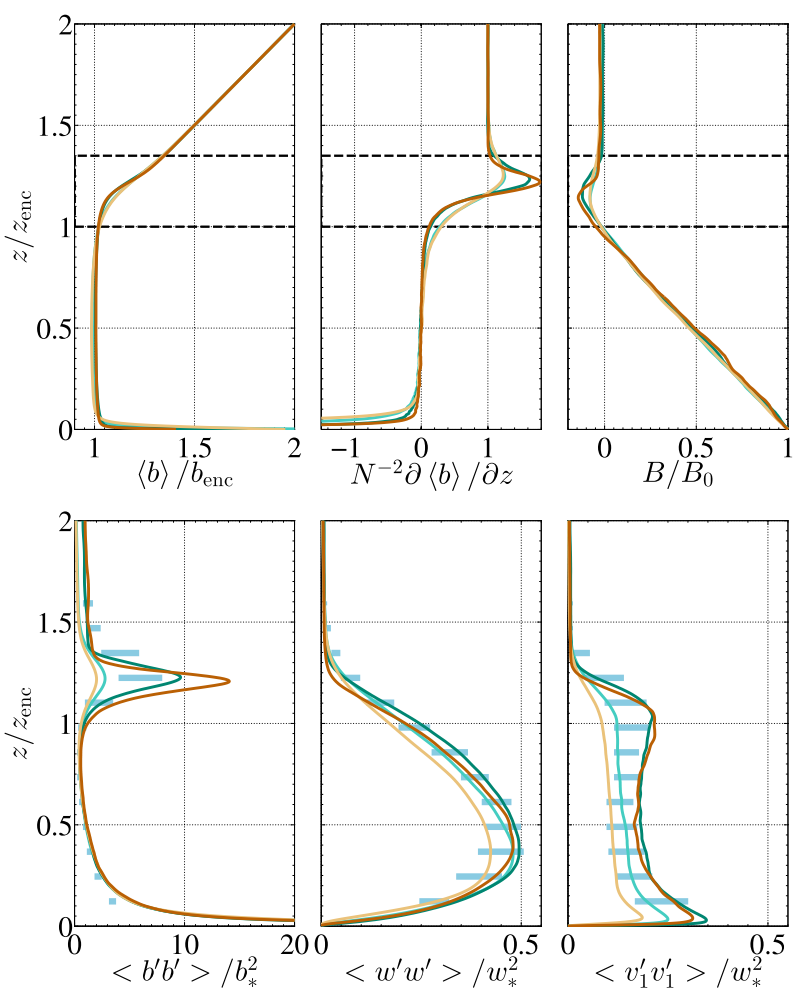

FIG. B1. (top, left to right) Normalized vertical profiles of the mean buoyancy $\langle b\rangle$, the mean buoyancy gradient $\partial\langle b\rangle / \partial z$, and the total mean buoyancy flux $B=\left\langle b^{\prime} w^{\prime}\right\rangle-\kappa \partial\langle b\rangle / \partial z$. (bottom, left to right) Normalized vertical profiles of the variance of the buoyancy and the vertical and horizontal velocities. Blue bars indicate the spread of LES data values at approximately $z_{\text {end }} / L_{0}=12$ from Fedorovich et al. (2004b). Blue indicates Re100 and brown indicates Re040. Light to dark colors indicate snapshots at $z_{\text {end }} / L_{0} \simeq$ $\{10,18\}$, respectively, for $\operatorname{Re} 100$. For $\mathrm{Re} 040$ case, snapshots at $z_{\text {end }}$ $L_{0} \simeq\{10,26\}$ are included.

are worth noting. First, the deviation of the mean buoyancy from the encroachment buoyancy, measured as $\langle b\rangle_{\min } / b_{\text {enc }}$, is very small, already less than $2 \%$ for $z_{\mathrm{end}} /$ $L_{0} \simeq 10$. Indeed, profiles of buoyancy-related quantities in Fig. B1 show self-similarity from $z_{\text {end }} / L_{0} \simeq 10$ onward. Second, the deviation of the vertical velocity fluctuation from scaling with the convective velocity is more significant at early times, but for the case Re100, the scaling with $w_{*}$ is already observed at $z_{\text {end }} / L_{0} \simeq 12$. Last, the deviation of the horizontal velocity fluctuation is most visible, since the scaling with $w_{*}$ is observed relatively later, roughly at $z_{\text {end }} / L_{0}>16$ for $\operatorname{Re} 040$ and $z_{\text {end }} / L_{0}>14$ for Re100. This slower development toward being proportional to $w_{*}$ is not a domain size effect, as shown in Fig. B2b by the collapse of the curves from the Re040 case and the $\mathrm{Re} 040 . \mathrm{R} 1$ case with the same $\mathrm{Re}_{0}$ but with a horizontal domain that is half as large in each horizontal direction (see Table B1).
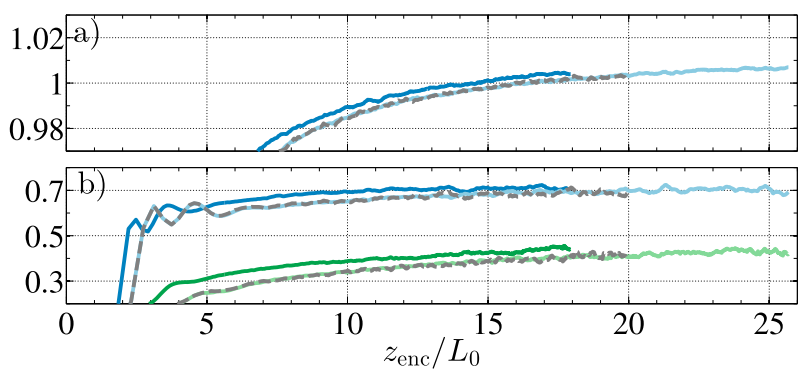

FIG. B2. (a) Temporal evolution of $\langle b\rangle_{\min } / b_{\text {enc }}$. (b) Temporal evolution of the normalized rms of vertical (blue) and horizontal (green) velocity fluctuations taken at the height of maximum $w_{\text {rms }}$. Average values calculated beyond $z_{\text {end }} / L_{0} \simeq 10$ are summarized in Table 1. Light colors correspond to Re040, dark colors correspond to Re100. Dashed lines correspond to Re040.R1, a simulation with a domain size that is half as large in each horizontal direction (Table B1).

The spread of values for the turbulent fluctuations from the LES intercomparison of Fedorovich et al. (2004b), taken at approximately $z_{\text {end }} / L_{0}=12$, is included in Fig. B1. This spread is comparable to the growth from $z_{\text {end }} / L_{0}=10$ to $z_{\text {end }} / L_{0}=26$ of the DNS data, and the variation of DNS data between the Re040 and Re100 cases at $z_{\text {end }} / L_{0} \simeq 10$. DNS data at the end of the simulation, when the Reynolds number is highest, is at the upper limit of the spread of LES values.

Whereas the encroachment and convective scales are appropriate characteristic scales within the mixed layer, they are clearly inappropriate scales within the entrainment zone for both the mean and the variance of buoyancy, respectively, since the normalized mean buoyancy gradient and the normalized buoyancy variance are increasing in time (Fig. B1). This behavior occurs for both Re040 and Re100 and has also been observed in LES data (Sorbjan 2007), which indicates a temporal evolution of the entrainment zone different from that of the mixed layer, rather than a low Reynolds number effect. We have provided in section 5 more appropriate scalings for these statistics inside the EZ.

TABLE B1. Simulations used in the study of the sensitivity of the results to the finite domain size and the grid resolution. Case Re040.R1 is equal to Case Re040 in Table 1, but with half the horizontal extension, i.e., $107 L_{0} \times 107 L_{0} \times 56.6 L_{0}$. Case Re040.R2 is equal to Case040.R1, but with twice the grid resolution (half the grid spacing in each of the three directions). The last column provides the resolution (in Kolmogorov units) at $z_{\text {end }} / L_{0} \simeq 13$.

\begin{tabular}{lccc}
\hline \hline Simulation & $\mathrm{Re}_{0}$ & Grid & $(\Delta z / \eta)_{\max }$ \\
\hline Re040.R1 & 42 & $1280 \times 1280 \times 576$ & 0.82 \\
Re040.R2 & 42 & $2560 \times 2560 \times 1152$ & 0.41 \\
\hline
\end{tabular}



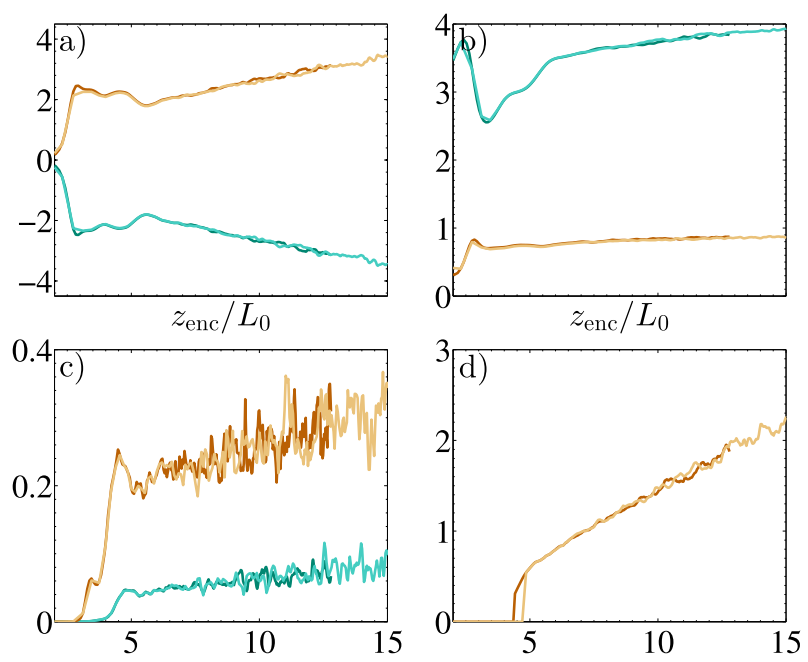

FIG. C1. Comparison between simulation Re040.R1 (light colors) and simulation Re040.R2 with double the spatial resolution (dark colors) used in the grid resolution study (Table B1). Statistics at the wall are as follows. (a) Normalized TKE budget: dissipation term $-\varepsilon_{\text {wall }} / B_{0}$ (blue); transport term $(-\partial T / \partial z)_{\text {wall }} / B_{0}$ (brown). (b) Normalized mean buoyancy difference $\left(\langle b\rangle_{\text {wall }}-b_{\text {enc }}\right) /\left(B_{0}^{3} / \kappa\right)^{1 / 4}$ (blue); normalized rms of the buoyancy fluctuation $b_{\mathrm{rms}} /\left(B_{0}^{3} / \kappa\right)^{1 / 4}$ (brown), where $\left(B_{0}^{3} / \kappa\right)^{1 / 4}$ is the diffusive buoyancy scale (see Mellado 2012). Statistics within the entrainment zone are as follows: (c) maximum of transport term $\max (-\partial T / \partial z) / B_{0}$ (brown), with turbulent contribution to the entrainment ratio $A_{t}$ (blue), and (d) normalized rms of the buoyancy fluctuation $b_{\mathrm{rms}} / b_{*}$.

\section{APPENDIX C}

\section{Grid Resolution Study}

We have quantified the dependence of the statistics on the grid resolution by comparing simulations Re040.R1 and Re040.R2 (see Table B1). The grid spacing in case $\mathrm{Re} 040 . \mathrm{R} 2$ is half the size of the grid spacing in case Re040. $\mathrm{R} 1$, and the corresponding initial conditions have been obtained by interpolating the initial fields from case Re040.R1 into the grid from case Re040.R2. The rest of the parameters in both simulations are exactly the same. We have measured the resolution in Kolmogorov units, $\Delta z / \eta$, as it is customary in DNS (Moin and Mahesh 1998; Pope 2000).

For the resolution $(\Delta z / \eta)_{\max } \simeq 1$ considered in this work, the average difference in the relevant statistics between cases Re040.R1 and Re040.R2, both in the near-wall region and in the entrainment zone, remains less than 3\% (Fig. C1), which is comparable to the statistical convergence that we achieve with the domain size considered in this work. This result, along with the scalings of the diffusive layer next to the wall and the Kolmogorov scale in terms of the kinematic viscosity $\nu$ and surface buoyancy flux $B_{0}$, allows us to estimate, for a given grid size, the maximum reference Reynolds number $\mathrm{Re}_{0}$ that still guarantees a good enough resolution of the small scales, when using the numerical scheme described in section 2. Further discussion can be found in Mellado (2012).

\section{REFERENCES}

Betts, A. K., 1974: Reply to comment on the paper "Non-precipitating cumulus convection and its parameterization." Quart. J. Roy. Meteor. Soc., 100, 469-471, doi:10.1002/qj.49710042517.

Carruthers, D. J., and J. C. R. Hunt, 1986: Velocity fluctuations near an interface between a turbulent region and a stably stratified layer. J. Fluid Mech., 165, 475-501, doi:10.1017/ S002211208600318X.

Carson, D., and F. Smith, 1975: Thermodynamic model for the development of a convectively unstable boundary layer. $A d$ vances in Geophysics, Vol. 18, Elsevier, 111-124, doi:10.1016/ S0065-2687(08)60455-0.

Chung, D., and G. Matheou, 2012: Direct numerical simulation of stationary homogeneous stratified sheared turbulence. J. Fluid Mech., 696, 434-467, doi:10.1017/jfm.2012.59.

Deardorff, J. W., 1970: Convective velocity and temperature scales for the unstable planetary boundary layer and for Rayleigh convection. J. Atmos. Sci., 27, 1211-1213, doi:10.1175/ 1520-0469(1970)027<1211:CVATSF >2.0.CO;2.

— G. E. Willis, and B. H. Stockton, 1980: Laboratory studies of the entrainment zone of a convectively mixed layer. J. Fluid Mech., 100, 41-64, doi:10.1017/S0022112080001000.

de Roode, S., P. Duynkerke, and H. Jonker, 2004: Large-eddy simulation: How large is large enough? J. Atmos. Sci., 61, 403421, doi:10.1175/1520-0469(2004)061<0403:LSHLIL>2.0.CO;2.

Dimotakis, P. E., 2000: The mixing transition in turbulent flows. J. Fluid Mech., 409, 69-98, doi:10.1017/S0022112099007946.

Driedonks, A. G. M., 1982: Models and observations of the growth of the atmospheric boundary layer. Bound.-Layer Meteor., 23, 283-306, doi:10.1007/BF00121117.

Fedorovich, E., and D. V. Mironov, 1995: A model for a shear-free convective boundary layer with parameterized capping inversion structure. J. Atmos. Sci., 52, 83-96, doi:10.1175/ 1520-0469(1995)052<0083:AMFASF>2.0.CO;2.

—, R. Conzemius, and D. Mironov, 2004a: Convective entrainment into a shear-free linearly stratified atmosphere: Bulk models reevaluated through large-eddy simulation. J. Atmos. Sci., 61, 281-295, doi:10.1175/1520-0469(2004)061<0281: CEIASL $>2.0 . \mathrm{CO} ; 2$.

— boundary layers as predicted by different large eddy simulation codes. Preprints, 16th Symp. on Boundary Layers and Turbulence, Portland, ME, Amer. Meteor. Soc., P4.7. [Available online at http://ams.confex.com/ams/pdfpapers/78656.pdf.]

Fernando, H. J. S., 1991: Turbulent mixing in stratified fluids. Annu. Rev. Fluid Mech., 23, 455-493, doi:10.1146/ annurev.fl.23.010191.002323.

— convection. Phys. Fluids, 2A, 1592-1596, doi:10.1063/1.857566.

Garratt, J. R., 1992: The Atmospheric Boundary Layer. Cambridge University Press, 316 pp.

Guillemet, B., H. Isaka, and P. Mascart, 1983: Molecular dissipation of turbulent fluctuations in the convective mixed layer. Part I: Height variations of dissipation rates. Bound.-Layer Meteor., 27, 141-162, doi:10.1007/BF00239611. 
Hebert, D. A., and S. M. de Bruyn Kops, 2006: Predicting turbulence in flows with strong stable stratification. Phys. Fluids, 18, 066602, doi:10.1063/1.2204987.

Hopfinger, E. J., 1987: Turbulence in stratified fluids: A review. $J$. Geophys. Res., 92 (C5), 5287-5303, doi:10.1029/JC092iC05p05287.

Jimenez, J., 2013: Near-wall turbulence. Phys. Fluids, 25, 101302, doi:10.1063/1.4824988.

Jonker, H. J. J., and M. A. Jimenez, 2014: Laboratory experiment of convective entrainment using saline water tank. Bound.Layer Meteor., 151, 479-500, doi:10.1007/s10546-014-9909-3.

—_, M. van Reeuwijk, P. Sullivan, and N. Patton, 2012: Interfacial layers in clear and cloudy atmospheric boundary layers. Proc. Seventh Int. Symp. on Turbulence, Heat and Mass Transfer, Palermo, Italy, International Centre for Heat and Mass Transfer, 3-14, doi:10.1615/ ICHMT.2012.ProcSevIntSympTurbHeatTransfPal.10.

Lilly, D. K., 1968: Models of cloud-topped mixed layers under a strong inversion. Quart. J. Roy. Meteor. Soc., 94, 292-309, doi:10.1002/qj.49709440106.

Linden, P. F., 1975: The deepening of a mixed layer in a stratified fluid. J. Fluid Mech., 71, 385-405, doi:10.1017/S0022112075002637.

Mahrt, L., 1979: Penetrative convection at the top of a growing boundary layer. Quart. J. Roy. Meteor. Soc., 105, 469-485, doi:10.1002/qj.49710544411.

Mellado, J. P., 2012: Direct numerical simulation of free convection over a heated plate. J. Fluid Mech., 712, 418-450, doi:10.1017/ jfm.2012.428.

— , and C. Ansorge, 2012: Factorization of the Fourier transform of the pressure-Poisson equation using finite differences in colocated grids. Z. Angew. Math. Mech., 92, 380-392, doi:10.1002/ zamm.201100078.

Moin, P., and K. Mahesh, 1998: Direct numerical simulation: A tool in turbulence research. Annu. Rev. Fluid Mech., 30, 539-578, doi:10.1146/annurev.fluid.30.1.539.

Monin, A. S., and A. M. Yaglom, 2007: Statistical Fluid Mechanics. Vol. 1, Mechanics of Turbulence, Dover, $769 \mathrm{pp}$.

Ozmidov, R. V., 1965: On the turbulent exchange in a stably stratified ocean. Izv. Atmos. Oceanic Phys., 1 (8), 853-860.

Pope, S. B., 2000: Turbulent Flows. Cambridge University Press, 771 pp.

Smyth, W. D., and J. N. Moum, 2000: Length scales of turbulence in stably stratified mixing layers. Phys. Fluids, 12, 1327-1342, doi:10.1063/1.870385.
Sorbjan, Z., 1999: Similarity of scalar fields in the convective boundary layer. J. Atmos. Sci., 56, 2212-2221, doi:10.1175/ 1520-0469(1999)056<2212:SOSFIT >2.0.CO;2.

- 2007: A numerical study of daily transitions in the convective boundary layer. Bound.-Layer Meteor., 123, 365-383, doi:10.1007/s10546-006-9147-4.

Stevens, B., and D. H. Lenschow, 2001: Observations, experiments, and large eddy simulation. Bull. Amer. Meteor. Soc., 82, 283-294, doi:10.1175/1520-0477(2001)082<0283:OEALES >2.3.CO;2.

Stull, R. B., 1973: Inversion rise model based on penetrative convection. J. Atmos. Sci., 30, 1092-1099, doi:10.1175/ 1520-0469(1973)030<1092:IRMBOP >2.0.CO;2.

Sullivan, P. P., and E. G. Patton, 2011: The effect of mesh resolution on convective boundary layer statistics and structures generated by large-eddy simulations. J. Atmos. Sci., 68, 23952415, doi:10.1175/JAS-D-10-05010.1.

_ C.-H. Moeng, B. Stevens, D. H. Lenschow, and S. D. Mayor, 1998: Structure of the entrainment zone capping the convective atmospheric boundary layer. J. Atmos. Sci., 55, 3042-3064, doi:10.1175/1520-0469(1998)055<3042:SOTEZC >2.0.CO;2.

Tennekes, H., 1975: Reply. J. Atmos. Sci., 32, 992-995, doi:10.1175/ 1520-0469(1975)032<0992:R>2.0.CO;2.

— Press, 300 pp.

Träumner, K., C. Kottmeier, U. Corsmeier, and A. Wieser, 2011: Convective boundary-layer entrainment: Short review and progress using Doppler lidar. Bound.-Layer Meteor., 141, 369391, doi:10.1007/s10546-011-9657-6.

Turner, J. S., 1973: Buoyancy Effects in Fluids. Cambridge University Press, $367 \mathrm{pp}$.

vanZanten, M., P. Duynkerke, and J. Cuijpers, 1999: Entrainment parameterization in convective boundary layers. J. Atmos. Sci., 56, 813828, doi:10.1175/1520-0469(1999)056<0813:EPICBL > 2.0.CO;2.

Xuequan, E., and E. Hopfinger, 1986: On mixing across an interface in stably stratified fluid. J. Fluid Mech., 166, 227-244, doi:10.1017/S0022112086000125.

Zeman, O., and H. Tennekes, 1977: Parameterization of the turbulent energy budget at the top of the daytime atmospheric boundary layer. J. Atmos. Sci., 34, 111-123, doi:10.1175/ 1520-0469(1977)034<0111:POTTEB > 2.0.CO;2.

Zilitinkevich, S. S., 1991: Turbulent Penetrative Convection. Avebury Technical, $179 \mathrm{pp}$. 